\title{
ON WALSH-FOURIER SERIES $\left({ }^{1}\right)$
}

\author{
BY \\ GEORGE W. MORGENTHALER
}

1. Introduction. In recent papers N. J. Fine $[1 ; 2 ; 3]\left({ }^{2}\right)$ has developed effective notation and techniques and has achieved important results in major areas of the Fourier theory of the Walsh orthonormal system which, is the completion of the following orthonormal system introduced by Rademacher [10] in 1922:

$$
\begin{aligned}
\phi_{0}(x) & =\left\{\begin{array}{rr}
1, & 0 \leqq x<1 / 2, \\
-1, & 1 / 2 \leqq x<1,
\end{array}\right. \\
\phi_{0}(x+1) & =\phi_{0}(x), \\
\phi_{n}(x) & =\phi_{0}\left(2^{n} x\right) .
\end{aligned}
$$

Fine's papers refer to earlier work on Fourier properties of Walsh series by Walsh, Kaczmarz, Steinhaus, and Paley. The latter's modified definition of the Walsh functions will be used here:

(a) $\psi_{0}(x) \equiv 1$,

(b) If $n$ has the unique dyadic expansion $\sum_{i=0}^{\infty} x_{i} 2^{i}$, where $x_{i}=0,1$ and $x_{i}=0$, for $i>m_{r}$, then $\psi_{n}(x)=\phi_{m_{1}}(x) \phi_{m_{2}}(x) \cdots \phi_{m_{i}}(x)$ where $m_{1}, \cdots, m_{r}$ corresponds to the coefficients $x_{m_{i}}=1$.

Every function $f(x)$ which is of period 1 and Lebesgue integrable on $[0,1]$ may be expanded in a Walsh-Fourier series $\left({ }^{3}\right), f(x) \sim \sum_{k=0}^{\infty} a_{k} \psi_{k}(x)$, where $a_{k}=\int_{0}^{1} f(x) \psi_{k}(x) d x, k=0,1,2, \cdots$.

Fine exhibited some of the basic similarities and differences between the trigonometric orthonormal system and the Walsh system. He identified the Walsh functions with the full set of characters of the dyadic group $G$. Contemporary with the work of Fine and somewhat more general is the work of

Presented to the Society, April 26, 1952 under the title Theorems on series of Walsh functions; received by the editors September 13,1954 and, in revised form, April 2, 1956 and May 3, 1956.

(1) This work, supported by the Office of Naval Research, forms the second part of a dissertation submitted in June, 1953 in partial fulfillment of the requirements for the Ph.D. degree at the University of Chicago. The author wishes to express his gratitude to Professor Antoni Zygmund for his valuable suggestions and encouragement. The referee also has been most helpful with suggestions for shortening proofs of several theorems, particularly those of $\$ 5$.

(2) The numbers in brackets refer to the bibliography at the end of the paper.

(3) Hereinafter "W.F.S." will denote "Walsh-Fourier series." 
$\mathrm{N}$. Vilenkin [17] on expansion of functions in Fourier series of the characters of an arbitrary zero-dimensional, separable group. A. A. Sneider $[13 ; 14 ; 15]$ applied this theory to obtain results on the Walsh system. Other results on Fourier properties of Walsh functions paralleling the classical trigonometric results have been reported in recent articles by Sunouchi [16] and Yano $[18 ; 19]$.

The object of the present study is to extend the comparison of trigonometric and Walsh series formed in the above papers. A summary of the principal results will appear presently, but first it is necessary to acquaint the reader with some of the main facts of the situation as found in Fine's work.

The dyadic group $G$ is the set of all sequences $\bar{x}=\left\{x_{n}\right\}, x_{n}=0,1, n$ $=1,2,3, \cdots$, the operation of $G$ being addition modulo 2 in each coordinate. Denote this addition in $G$ by " + " and let the identity of elements in $G$ be denoted by " =".

Corresponding to each element $\bar{x}=\left\{x_{n}\right\}$ of $G$ there is a real number

$$
\lambda(\bar{x})=\frac{x_{1}}{2}+\frac{x_{2}}{2^{2}}+\frac{x_{3}}{2^{3}}+\cdots
$$

lying in the closed interval $[0,1]$. At the dyadic rationals $x: 0<x<1$ we have two representations in the dyadic scale, and hence two elements of $G$ map onto these. If $\mu(x)$ is the inverse of $\lambda$ we have for all real $x$

$$
\lambda(\mu(x))=x-[x]
$$

the finite expansion in $G$ being associated under $\mu$ with dyadic rationals. Then $\mu(\lambda(\bar{x}))=\bar{x}$ provided $\lambda(\bar{x})$ is not a dyadic rational.

Fine establishes that $\phi_{n-1}(x)=\chi_{n}(\mu(x)), n \geqq 1$, where $\chi_{n}(\bar{x})=\chi_{n}\left(\left\{x_{1} x_{2} \cdots\right\}\right)$ is a character of $G$ defined as +1 if $x_{n}=0 ;-1$ if $x_{n}=1$. All characters of $G$ may be obtained by taking finite products of these basic characters. Each Walsh function is expressible as $\psi_{n}(x)=\chi_{j_{1}}(\mu(x)) \chi_{j_{2}}(\mu(x)) \cdots \chi_{j_{t}}(\mu(x))$, and so the Walsh functions are identified with the full set of characters of $G$.

For the convenient abbreviation

$$
\lambda(\mu(y) \stackrel{*}{+} \mu(z))=y+z,
$$
$y, z$ real,

several useful relations have been established. In particular,

$$
\text { (a) } x+y=\sum_{n=1}^{\infty} \frac{\left|x_{n}-y_{n}\right|}{2^{n}} \text { where } x=\sum_{n=1}^{\infty} \frac{x_{n}}{2^{n}}, y=\sum_{n=1}^{\infty} \frac{y_{n}}{2^{n}},
$$

finite expansions being used for dyadic rationals.
(b) $|(x+h)-x| \leqq h$,
$0 \leqq x<1,0 \leqq h<1$
(c) $\psi_{k}(x+y)=\psi_{k}(x) \psi_{k}(y)$ whenever $(x+y)$ is not a dyadic rational; this exception is a denumerable event for each fixed $x$. 
A basic property of the abbreviation " $\dot{+}$ " is the invariance of the Lebesgue integral with respect to it. That is, let $x$ be fixed, let $y$ belong to a measurable set $A$ lying in the unit interval, and let $T_{x}(A)=\{(x+y) \mid y \in A\}$. Then $\left.\left|T_{x}(A)\right|=|A|{ }^{4}\right)$. If $f(x)$ is integrable, then for every fixed $x$,

$$
\int_{0}^{1} f(x+y) d y=\int_{0}^{1} f(y) d y .
$$

There is a natural topology of $G$ which is obtained by taking as neighborhoods the sets of points $\left\{x_{1}, x_{2}, \cdots, x_{n}, d_{n+1} \cdots\right\}$ in which $x_{1}, \cdots, x_{n}$ are fixed and $d_{n+1}, \cdots$ vary independently. These neighborhoods, $N\left(x_{1}, x_{2}, \cdots\right.$, $\left.x_{n}\right)$, form a basis for $G . \lambda: G \rightarrow R[0,1]$ defines a metric on $G$ which is continuous on $G$, and the topology induced on $G$ by this metric is equivalent to the original topology.

To each real-valued function $g(x)$ of period 1 there corresponds a function $\bar{g}(\bar{x})$ on $G$ defined by

$$
\begin{aligned}
& \bar{g}(\bar{x})=g(x) \text { if } \mu(x)=\bar{x} \text { for some } x \in[0,1], \\
& \bar{g}(\bar{x})=\limsup _{y \rightarrow x} \bar{g}(\bar{y}) \text { if } \mu(x) \neq \bar{x} \text { for any } x \in[0,1]
\end{aligned}
$$

where lim sup is taken over those $\bar{y}$ which corresond to dyadic irrationals. This definition forms the basis for discussing the relation between two given classes of functions, one class on $G$ and one on $[0,1]$.

Characteristic functions of neighborhoods of $G$ are continuous on $G$ since each neighborhood is both open and closed. Finite linear combinations of such characteristic functions are then continuous on $G$.

In $\$ 2$ the Haar measure on $G$ is exhibited, and the classes of measurable and integrable real-valued functions on $G$ are related to the corresponding classes on $[0,1]$.

$\$ 3$ deals with order of coefficients and investigates further the startling result of Fine that, unlike the trigonometrical case, the only absolutely continuous functions whose Walsh-Fourier coefficients satisfy $b_{k}=o(1 / k)$ are the constants. It is shown in particular that the arithmetic mean of the sequence $\left\{k\left|b_{k}\right|\right\}$ does tend to zero. As a subsidiary result, the class of functions on $[0,1]$ whose $G$-extensions are continuous with respect to the topology of $G$ is characterized.

The class of functions on $[0,1]$ whose $G$-extensions are Lipschitz functions on $G$ is identified in $\$ 4$, and analogues of various results from the trigonometric Fourier theory of Lipschitz $\alpha$ functions are obtained. The analogues of the well known theorems of Rogosinski are established in $\S 5$. The most interesting result of this section is the verification of the formula

$$
f(x)=\frac{1}{2}\left\{\liminf _{n \rightarrow \infty} S_{n}(x)+\underset{n \rightarrow \infty}{\lim \sup } S_{n}(x)\right\} \text { a.e. on }[0,1]
$$

(4) $|E|$ denotes the Lebesgue measure of the set $E$. 
when $f(x)$ is integrable and the partial sums $S_{n}(x)$ of the W.F.S. for $f(x)$ satisfy $\lim \sup _{n \rightarrow \infty} S_{n}(x)<+\infty$. $\S 6$ establishes the analogues of some results of the transformation theory of trigonometric series.

In $\$ 7$ on lacunary Walsh series, the Walsh analogue of the Central Limit Problem of Salem and Zygmund [12] is established. Another well known property of trigonometrical lacunary series is established for the Walsh functions: if the partial sums $S_{N}(x)$ of a lacunary Walsh series oscillate finitely at each point of an interval, then $\sum_{k=1}^{\infty}\left|a_{k}\right|<+\infty$. This section also establishes the Walsh analogues of the theorems of Banach on the existence of Fourier series with given coefficients at prescribed lacunary places.

In the final section an example is given of a continuous nondecreasing function whose Walsh Fourier-Stieltjes coefficients do not tend to zero.

2. Haar measure on $G$. The discussion of the Fourier properties of the Walsh system may proceed from two points of view:

(a) Characters $\chi_{n}(x)$ of $G$ and their products, Haar measure on $G$,

The various classes of functions on $G$,

or

(b) Functions $\psi_{n}(x)$ on $[0,1]$, Lebesgue measure on $[0,1]$, The various classes of functions on $[0,1]$, usually related to the classes on $G$ in some sense involving definition (1.8).

The work of Fine on W.F.S. followed mainly the second approach, reference being made to $G$ only when a particular property of $\dot{+}$ was required. Because of the analogy with the exponentials $\left\{e^{2 \pi i n x}\right\}$, it may be thought that the first approach is preferable. The present study shall attempt an analysis which permits both points of view.

Letting $m$ denote the obvious product measure on $G$ and $\bar{m}$ its completion (see Halmos [4, pp. 158-159]), the following theorem is immediate.

Theorem I. (1) If $f(x)$ is Borel (Lebesgue) measurable on $[0,1]$, its $G$ extension by (1.8), $\bar{f}(\bar{x})$, is Borel $(\bar{m})$ measurable on $G$ and conversely.

(2) If $f(x)$ is Lebesgue integrable on $[0,1]$, then the G-extension, $\bar{f}(\bar{x})$, is integrable on $G$ with respect to $\bar{m}$ and conversely, the integrals being equal.

This theorem essentially implies that the two basic points of view listed above should yield the same Fourier results with respect to classes of functions characterized by integrability conditions.

3. On the order of coefficients. Fine has obtained the classical results for the order of Walsh-Fourier coefficients of various classes of functions. However, an important difference was displayed in the case of coefficients of absolutely continuous functions. If $f(x)$ has mean value zero over $(0,2 \pi)$, the periodic function $F(x)=\int_{0}^{x} f(t) d t$ has trigonometric Fourier coefficients which 
are $o(1 / k)$. Fine showed that the only absolutely continuous functions on $[0,1]$ whose Walsh-Fourier coefficients are $o(1 / k)$ are constants. The following theorem shows that "on the average" the coefficients behave as they do in the classical system.

THEOREM II. Let $f(x)$ be real-valued, periodic, and of mean value zero on $[0,1]$. Then if $F(x)=\int_{0}^{x} f(t) d t$ and $F(x) \sim \sum_{k=0}^{\infty} b_{k} \psi_{k}(x)$ the arithmetic means of the sequence $\left\{k\left|b_{k}\right|\right\}$ tend to zero.

Proof. Let $f(x) \sim \sum_{k=1}^{\infty} a_{k} \psi_{k}(x)$. Then by Fine [1, Theorem VII] for fixed $k^{\prime} \geqq 0$ and $\nu \rightarrow \infty$,

$$
b_{2^{n}+k^{\prime}}=-2^{-(n+2)} a_{k^{\prime}}+o\left(2^{-n}\right) .
$$

It follows that $k\left|b_{k}\right| \leqq\left|a_{k}{ }^{\prime}\right|+o(1)$, where $k=2^{\nu}+k^{1}, k^{1}<2^{\nu}$. Therefore

$$
\begin{aligned}
\frac{1}{n+1} \sum_{k=0}^{n} k\left|b_{k}\right| & \leqq \frac{1}{n+1} \sum_{0 \leqq k \leqq n ; k^{\prime} \leqq T}\left|a_{k^{\prime}}\right|+\frac{1}{n+1} \sum_{0 \leqq k \leqq n ; k^{\prime}>T}\left|a_{k^{\prime}}\right|+o(1) \\
& =S_{1}+S_{2}+o(1) .
\end{aligned}
$$

Given $\epsilon>0$, choose $T$ so that $\left|a_{k^{\prime}}\right|<\epsilon$ for $k^{\prime}>T$. Then $S_{2}<\epsilon$. For $S_{1}$ write $k=2^{\nu}+k^{\prime}$, where $0 \leqq \nu \leqq r, 0 \leqq k^{\prime} \leqq T$, and $r$ is defined by $2^{r} \leqq n<2^{r+1}$. Then

$$
S_{1} \leqq \frac{1}{n+1} \sum_{r=0}^{r} \sum_{k^{\prime}=0}^{T}\left|a_{k^{\prime}}\right| \leqq M \frac{\left(1+\log _{2} n\right)}{n+1}=o(1)
$$

and the proof is complete.

Imposing a still stronger smoothness condition on a function is reflected, in the trigonometric system, in a more rapid convergence of the Fourier coefficients to zero. Specifically, if $F(x)$ is $p$ times differentiable, its Fourier coefficients $\left\{b_{k}\right\}$ satisfy $k^{p}\left|b_{k}\right| \rightarrow 0$. Once again the jumps of the Walsh functions cannot be smoothed out in time, and the corresponding property is lacking entirely. In fact, the damage is so thorough that a strongly negative result similar to the result of Fine can be established. The proof will not be given here $\left({ }^{5}\right)$.

THEOREM III. The only twice-differentiable functions $F(x), \quad F(x)$ $\sim \sum_{k=0}^{\infty} b_{k} \psi_{k}(x)$ for which the arithmetic means of the sequence $\left\{k^{2}\left|b_{k}\right|\right\}$ tend to zero are the constants.

A well known result from trigonometric Fourier series is the proposition that if the Fourier coefficients of a function $f(x)$ of bounded variation are $o(1 / n), f(x)$ is continuous. This result is not valid for the Walsh functions. Walsh polynomial coefficients, being zero from some place on, are surely $o(1 / n)$, however, such polynomials are not continuous functions. If continuity in the group $G$ is considered, a partial analogue may be obtained. It is con-

${ }^{(5)}$ Where proofs are not given, they are found in the author's thesis (see footnote 1 ). 
venient first to define a special class of functions on $[0,1]$ which are related to the continuous functions on $G$.

Recalling the meaning of the induced combining symbol " $\dot{+}$ " on the interval $[0,1]$ given in (1.5), we make the following definition:

A function $f(x)$ of period 1 is "continuous $(W)$ " at a point $x$ of $0 \leqq x<1$ if $\epsilon>0 \cdot \supset \cdot \exists \delta(\epsilon, x)>0 \ni|f(x+y)-f(x)|<\epsilon$ whenever $0 \leqq y<\delta(\epsilon, x)$.

Any continuous function is continuous $(W)$. The usual algebraic properties of continuous functions hold for continuous $(W)$ functions. Any Walsh function is continuous $(W)$, for, at their dyadic rational discontinuities $x_{0}$, $\left(x_{0}+y\right)$ lies to the right of $x_{0}$ for $y$ sufficiently small, and Walsh functions are continuous on the right. Consider $f(x)=\sum_{d_{i} \leqq x} \delta_{i}$, where $\left\{d_{i}\right\}$ is an enumeration of the dyadic rationals in $[0,1]$ and $\delta_{i}>0, \sum \delta_{i}<+\infty$. This function is continuous $(W)$, not continuous, and is not a polynomial in Walsh functions.

The next theorem is easily verified and shows that the concept "continuous $(W)^{n}$ is a natural candidate to replace ordinary continuity on the real line in situations involving Walsh functions and the ordinary processes of analysis.

THEOREM IV. If $f(x)$ is continuous $(W)$ on $0 \leqq x<1$ and if $f(x-0)$ exists and is finite at each dyadic rational $x, 0<x \leqq 1$, then $\bar{f}(\bar{x})$, the G-extension of $f(x)$, is continuous on $G$. If $\bar{f}(\bar{x})$ is continuous on $G$, the function $f(x)=\bar{f}(\mu(x))$ is continuous $(W)$ on $0 \leqq x<1$ and $f(x-0)$ exists and is finite at each dyadic rational $0<x \leqq 1$.

To verify that in the theorem the condition " $f(x-0)$ exists and is finite at each dyadic rational $x$," is not redundant, it is enough to exhibit a function which is continuous $(W)$ but such that $f(x-0)=+\infty$ at a dyadic rational. The function $1 / 1-2 x$ on $0 \leqq x<1 / 2$ and equal to 1 on $1 / 2 \leqq x \leqq 1$ is such a function with $f(1 / 2-0)=+\infty$.

Theorem V. If $f(x)$ is nondecreasing and bounded on $0 \leqq x \leqq 1$ and its Walsh-Fourier coefficients satisfy $a_{2^{n}}=o\left(1 / 2^{n}\right)$, then the function $f^{*}(x)=f(x+0)$ has the same coefficients as $f(x)$ and has its $G$-extension continuous on $G$.

In view of the result of Fine, that the only absolutely continuous functions whose Walsh-Fourier coefficients are $o(1 / n)$ are the constants, it is well to observe that any nondecreasing step-function with dyadic intervals of constancy is an example of a function satisfying the conditions of the theorem, although it is not known how general such functions can be.

Proof. $f^{*}(x)$ obviously has the same coefficients as $f(x)$ and it is enough to show that $f^{*}$ has only dyadic rational discontinuities.

Lemma I. The Walsh-Fourier coefficients $\left\{a_{2^{n}}\right\}$ of the function

$$
g_{x}(t)=\left\{\begin{array}{lr}
0, & 0 \leqq t<x, \\
b, & x \leqq t<1, b>0
\end{array}\right.
$$


are nonpositive, zero for $x=m / 2^{r}$ and $n$ large, negative and $\neq o\left(1 / 2^{n}\right)$ for other $x$.

Proof. $a_{2^{n}}=b \int_{x}^{1} \psi_{2^{n}}(t) d t=-b J_{2^{n}}(x)=-b / 2^{n} J_{1}\left(2^{n} x\right)$ by Fine [1, p. 400]. If $x=m / 2^{r}$ then $a_{2^{n}}=0$ for $n \geqq r$ and is negative for $n<r$. If $x$ is not a dyadic rational the fractional part of $2^{n} x$ falls between $1 / 4$ and $3 / 4$ for infinitely many $n$.

Decompose $f^{*}(x)$ into a continuous part and a jump function, $f^{*}(x)$ $=g(x)+j(x)$. Clearly $j(x)=\sum_{0<c_{i} \leqq x}\left[f^{*}\left(c_{i}\right)-f^{*}\left(c_{i}-0\right)\right]$, where $\left\{c_{i}\right\}$ are the discontinuities of $f^{*}$. It is enough to show that $j(x)$ has no discontinuities other than dyadic rationals.

As in Lemma I, we denote the step-function at $c_{i}$ with jump $\left(f^{*}\left(c_{i}\right)\right.$ $\left.-f^{*}\left(c_{i}-0\right)\right)$ by $g_{c_{i}}(x)$. Then $j(x)=\lim _{N \rightarrow \infty} \sum_{i=1}^{N} g_{c_{i}}(x)$ and the value of the $2^{n}$ th Fourier coefficient of $j(x), a_{2^{n}}^{(j)}$, is $\sum_{i=1}^{\infty} a_{2^{n}}^{\left(c_{i}\right)}$, where $a_{2^{n}}^{\left(c_{i}\right)}$ is the $2^{n}$ th WalshFourier coefficient of $g_{c_{i}}(x)$.

Suppose $c_{i}$ is a dyadic irrational discontinuity of $j(x)$. Then by Lemma I,

$$
a_{2^{n}}^{(j)} \leqq a_{2^{n}}^{\left(c_{i}\right)} \leqq 0, \quad\left|a_{2^{n}}^{(j)}\right| \geqq\left|a_{2^{n}}^{\left(c_{i}\right)}\right|, \quad n=1,2,3, \cdots
$$

and

$$
a_{2^{n}}^{\left(c_{i}\right)} \neq o\left(1 / 2^{n}\right)
$$

However, the $2^{n}$ th Walsh-Fourier coefficients of $f^{*}(x)$ are $o\left(1 / 2^{n}\right)$. If the $2^{n}$ th Walsh-Fourier coefficient of the continuous part of $f^{*}(x), a_{2^{n}}^{(0)}$, is nonpositive, then $a_{2^{n}}=a_{2^{n}}^{(0)}+a_{2^{n}}^{(j)} \leqq a_{2^{n}}^{(j)} \leqq 0$, and hence $a_{2^{n}}^{(j)}=o\left(1 / 2^{n}\right)$, which would be a contradiction. But

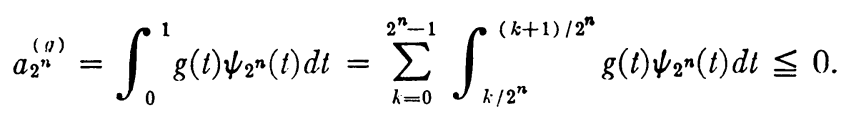

Each term in the sum is nonpositive since $g(x)$ is nondecreasing and $\psi_{2^{n}}$ is +1 on the first half of $\left[k / 2^{n},(k+1) / 2^{n}\right]$ and -1 on the second half. The theorem is therefore proved.

4. On convergence and summability. The $n$th partial sum and $n$th $(C, 1)$ mean of a Fourier series are denoted respectively by

$$
S_{n}(x ; f)=\sum_{k=0}^{n-1} a_{k} \psi_{k}(x) \quad \text { and } \quad \sigma_{n}(x ; f)=\frac{\sum_{k=1}^{n} S_{k}(x ; f)}{n} .
$$

The Dirichlet kernel is $D_{n}(x)=\sum_{k=0}^{n-1} \psi_{k}(x)$, and Fine has shown that $S_{n}(x)$ may be written

$$
S_{n}(x)=\int_{0}^{1} f(x+t) D_{n}(t) d t=\int_{0}^{1} f(t) D_{n}(x+t) d t .
$$

The size of $D_{n}(t)$ is estimated by $\left|D_{n}(t)\right|<2 / t, 0<t<1$; and $D_{2^{n}}(t)=2^{n}$ for $t \in\left[0,1 / 2^{n}\right)$ and zero elsewhere. 
Fejér's kernel is $K_{n}(x)=n^{-1} \sum_{k=1}^{n} D_{k}(x)$, so that

$$
\sigma_{n}(x)=\int_{0}^{1} f(x+t) K_{n}(t) d t .
$$

These formulas and other relations for $D_{n}(x)$ and $K_{n}(x)$ have obvious counterparts on $G$. For example, if $\Delta_{i}(\bar{x})$ represents the $i$ th character of $G\left(\Delta_{i}\right.$ being a finite product of the $\chi_{n}(\bar{x})$ ), then corresponding to (4.2)

$$
\bar{S}_{n}(\bar{x})=\int_{G} \bar{f}(\bar{y})\left(\sum_{i=0}^{n-1} \Delta_{i}(\bar{x} \stackrel{*}{+} \bar{y})\right) d \bar{m}=\int_{G} \bar{f}(\bar{x} \stackrel{*}{+} \bar{y}) \bar{D}_{n}(\bar{y}) d \bar{m} .
$$

Fine [3] has recently proved that if $f \in L(0,1)$, its W.F.S. is $(c, \alpha)$ summable a.e. $(\alpha>0)$. The following lemma due to Yano [18] is used in proving Theorem VI.

Lemma II. $\int_{0}^{1}\left|K_{n}(t)\right| d t \leqq 2, n=1,2,3, \cdots$.

Theorem VI. If $f(x) \in L(0,1), \int_{0}^{1}\left|\sigma_{k}(x ; f)-f(x)\right| d x \rightarrow 0$ as $k \rightarrow \infty$ where $\sigma_{k}(x ; f)$ is the $(C, 1)$ mean of the W.F.S. of $f(x)$. Similarly, $\int_{G}\left|\bar{\sigma}_{k}(\bar{x} ; \bar{f})-\bar{f}(\bar{x})\right| d \bar{m}$ $\rightarrow 0$ as $k \rightarrow \infty$ for every $\bar{f}$ in $L(G)$.

Proof. The proof of the last sentence of the theorem follows from the first sentence by Theorem I. Let $g(x)$ be a continuous function for which

$$
\int_{0}^{1}|f(x)-g(x)| d x<\epsilon .
$$

For continuous $g(x)$ the theorem is true since $\sigma_{k}(x ; g) \rightarrow g(x)$ uniformly (cf. Fine [1, Theorem 24 II]). Then

$$
\begin{aligned}
\int_{0}^{1}\left|\sigma_{k}(f)-f\right| d x \leqq & \int_{0}^{1}\left|\sigma_{k}(f)-\sigma_{k}(g)\right| d x+\int_{0}^{1}\left|\sigma_{k}(g)-g\right| d x \\
& +\int_{0}^{1}|f-g| d x \\
& \leqq \int_{0}^{1}\left|\sigma_{k}(f-g)\right| d x+o(1)+\int_{0}^{1}|f-g| d x \\
\leqq & 3 \int_{0}^{1}|f-g| d x+o(1), \text { since for any } h(x) \in L, \\
\int_{0}^{1}\left|\sigma_{k}(x ; h)\right| d x & =\int_{0}^{1}\left|\int_{0}^{1} h(x+t) K_{n}(t) d t\right| d x \\
\leqq & \int_{0}^{1}|h(x)|\left(\int_{0}^{1}\left|K_{n}(x+t)\right| d t\right) d x \leqq 2 \int_{0}^{1}|h| d x .
\end{aligned}
$$


This proves the theorem. The result may also be obtained from the work of Orlicz [8] on the mean convergence of Toeplitz transformations whose kernels are bounded. The idea of Orlicz applied to the Walsh case is to prove that $\int_{0}^{1}\left|\sigma_{k}(x ; f)\right| d x \leqq C \int_{0}^{1}|f(x)| d x$ (a result which is valid for $C=2$ ) for any integrable $f(x)$. This together with the point-wise convergence a.e. of $\sigma_{k}(x)$ $\rightarrow f(x)$ for $f(x)$ bounded implies the result.

We now consider certain problems involving the concept of a Lipschitz $\alpha$ class of functions, $0<\alpha \leqq 1$. Fine [1] has demonstrated, just as in the trigonometric case, that the W.F.S. of such functions converge uniformly to $f(x)$. Moreover, if $\alpha>1 / 2$, the series converges absolutely. Yano [19] has demonstrated that if $f(x)$ is a Lipschitz $\alpha$ function on $(0,1), 0<\alpha<1$, the $(C, 1)$ means of the W.F.S. for $f(x)$ satisfy $\sigma_{n}(x)-f(x)=O\left(n^{-\alpha}\right)$. Additional exact analogues of several results of trigonometrical Fourier theory (cf. Zygmund $[21$, p. 106]) can be obtained if the concept of a Lipschitz $\alpha$ function is carried to the group $G$.

We define the class of Lipschitz $\alpha$ functions $\bar{f}(\bar{x})$ on $G, 0<\alpha \leqq 1$, as those functions satisfying

$$
|\bar{f}(\bar{x})-\bar{f}(\bar{y})|<C \lambda(\bar{x} \stackrel{*}{+} \bar{y})^{\alpha} \text {, for some constant } C .
$$

We further define $f(x)$ on $[0,1]$ to be Lipschitz $\alpha(W), 0<\alpha \leqq 1$, if

$$
\begin{aligned}
& |f(x+y)-f(x)|<C y^{\alpha} \quad 0 \leqq x<1,0 \leqq y<1, \\
& \mu(x+y)=\mu(x) \stackrel{*}{+} \mu(y),
\end{aligned}
$$

i.e., except when $(\mu(x) \stackrel{*}{+} \mu(y))$ ends in a sequence of 1 's. The following theorem may then be established.

Theorem VII. If $\bar{f}(\bar{x})$ is a Lipschitz $\alpha$ function on $G, 0<\alpha \leqq 1$, then $f(x)$ $=\bar{f}(\mu(x))$ is Lipschitz $\alpha(W)$ function on $[0,1)$, and $f(x-0)$ exists and is finite at each dyadic rational $0<x \leqq 1$. If $f(x)$ is a Lipschitz $\alpha(W)$ function on $[0,1)$, $0<\alpha \leqq 1$, and $f(x-0)$ exists and is finite at each dyadic rational $0<x \leqq 1$, then the G-extension of $f, \bar{f}(\bar{x})$, is a Lipschitz $\alpha$ function on $G$.

REMARKs. (1) If $f(x)$ is a function of class Lipschitz $\alpha$ on $[0,1], f(x)$ is a Lipschitz $\alpha(W)$ function.

(2) If $f(x)$ is a function of class Lipschitz $\alpha(W)$ on $[0,1], f(x)$ is continuous $(W)$ on $[0,1]$.

(3) In the case of the system $\left\{e^{2 \pi i n x}\right\}$, each function is of Lipschitz class 1 , but the corresponding constant of the definition changes with each $n$. The $G$-extensions of the Walsh functions have a similar property. The function $\chi_{n}(\bar{x})$ is constant on each of the $2^{n}$ neighborhoods $N_{i}=N\left(x_{1}, x_{2}, \cdots, x_{n}\right)$. If $\bar{x}$ and $\bar{y}$ are in $N_{i},\left|\chi_{n}(\bar{x})-\chi_{n}(\bar{y})\right|=0$. If $\bar{x} \in N_{i}, \bar{y} \in N_{j}, i \neq j$, then

$$
\lambda(\bar{x} \stackrel{*}{+} \bar{y}) \geqq 1 / 2^{n}
$$


since the first $n$ coordinates of $\bar{x}$ must differ from the first $n$ coordinates of $\bar{y}$ in at least one place. Under all circumstances $\left|\chi_{n}(\bar{x})-\chi_{n}(\bar{y})\right| \leqq 2$, and it follows that for $C=2^{n+1}$,

$$
\left|\chi_{n}(\bar{x})-\chi_{n}(\bar{y})\right| \leqq C \lambda(\bar{x} \stackrel{*}{+} \bar{y}), \quad \bar{x}, \bar{y} \text { in } G .
$$

From these remarks it is easy to see that no constant will serve for every $n$. The statement is proved in the same way for a general character of $G$ which is a finite product of the $\chi_{n}(\bar{x})$. The function $\psi_{n}(x)$ is Lipschitz $\alpha(W)$ on $[0,1)$, and again the constant increases with $n$.

If $f(x)$ is a Lipschitz $\alpha$ function on $[0,1]$, then $\omega(\delta, f) \leqq C \delta^{\alpha}$ for some constant $C$ where $\omega(\delta, f)$ is the modulus of continuity of $f(x)$. We define

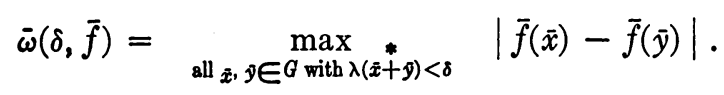

Clearly if $\bar{f}(\bar{x})$ is a Lipschitz $\alpha$ function on $G, \bar{\omega}(\delta, \bar{f}) \leqq C \delta^{\alpha}$. The counterpart of $\bar{\omega}(\delta, \bar{f})$ on the real line is

$$
\omega_{W}(\delta, f)=\underset{0 \leqq x<1 ; 0 \leqq y<\delta ;(\mu(x)+\mu(y)) \text { not ending in 1's }}{\max }|f(x+y)-f(x)| .
$$

If $f(x)$ is a Lipschitz $\alpha(W)$ function, $\omega_{W}(\delta, f)<C \delta^{\alpha}$. By Theorem VII, if $\bar{f}(\bar{x})$ is Lipschitz $\alpha$ on $G$, then $f(x)=\bar{f}(\mu(x))$ satisfies $\omega_{W}(\delta, f)<C \delta^{\alpha}$ on $[0,1]$.

The next results are stated both on $G$ and on $[0,1]$, but are proved on $G$.

TheOREM VIII. (1) If $\bar{f}(\bar{x})$ is a Lipschitz $\alpha$ function on $G$, then the partial sums $\bar{S}_{n}(\bar{x})$ of the Fourier development of $\bar{f}(\bar{x})$ relative to the characters of $G$ satisfy

$$
\bar{\omega}\left(\delta, \bar{S}_{n}(\bar{x})\right)=O\left(\delta^{\alpha} \log \frac{1}{\delta}\right) \text { uniformly in } n .
$$

(2) If $f(x)$ is a Lipschitz $\alpha(W)$ function on $[0,1]$ and $f(x-0)$ exists and is finite at dyadic rationals, then

$$
\omega_{W}\left(\delta, S_{n}(x)\right)=O\left(\delta^{\alpha} \log \frac{1}{\delta}\right) \text { uniformly in } n .
$$

Proof. For any $\bar{x}, \bar{y} \in G$ with $\lambda(\stackrel{*}{*}+\bar{y})<\delta$

$$
\begin{aligned}
\left|\bar{S}_{n}(\bar{x})-\bar{S}_{n}(\bar{y})\right| & =\left|\int_{G}[\bar{f}(\bar{x} \stackrel{*}{+} \bar{t})-f(\bar{y} \stackrel{*}{+} \bar{t})] \bar{D}_{n}(\bar{t}) d \bar{m}\right| \\
& \leqq\left|\int\right|_{\text {al1 } \bar{z} ; \lambda(\bar{l}) \leqq \delta}+\left|\int\right|_{\text {al1 } \bar{l} ; \lambda(\bar{l})>\delta .}
\end{aligned}
$$

The second integral (since $\lambda\left[\left(\bar{x}_{+}^{*} \bar{t}\right) \stackrel{*}{+}(\bar{y} \stackrel{*}{+} \bar{t})\right]=\lambda(\stackrel{*}{+}+\bar{y})<\delta$ ) satisfies 


$$
\begin{aligned}
\left|\int_{\mathrm{a} 11 \bar{i} ; \lambda(\bar{t})>\delta}\right| & \leqq C \delta^{\alpha} \int_{\mathrm{a} 11 \bar{l} ; \lambda(\bar{t})>\delta}\left|\bar{D}_{n}(\bar{t})\right| d \bar{m} \\
& \leqq C \delta^{\alpha} \int_{\delta}^{1} \frac{2}{t} d t=2 C \delta^{\alpha} \log \frac{1}{\delta} .
\end{aligned}
$$

For the first integral in (4.8) we have

$$
\begin{aligned}
& \left|\int_{\text {all } \overline{l ; \lambda}(\bar{l}) \leqq \delta}\left[\bar{f}\left(\bar{x}+{ }^{*} \bar{t}\right)-\bar{f}\left(\bar{y}+{ }^{*} \bar{t}\right)\right] \bar{D}_{n}(\bar{t}) d \bar{m}\right| \\
& \leqq \int_{\lambda(\bar{l}) \leqq \delta}|\bar{f}(\bar{x} \stackrel{*}{+} \bar{t})-\bar{f}(\bar{x})|\left|\bar{D}_{n}(\bar{t})\right| d \bar{m} \\
& +\int_{\lambda(\bar{l}) \leqq \delta}|\bar{f}(\bar{y})-\bar{f}(\bar{y}+* \hat{t})|\left|\bar{D}_{n}(\bar{t})\right| d \bar{m}+\left|\int_{\lambda(\bar{t}) \leqq \delta}[\bar{f}(\bar{x})-\bar{f}(\bar{y})] \bar{D}_{n}(\bar{t}) d \bar{m}\right| .
\end{aligned}
$$

But since $\lambda((\bar{x} \stackrel{*}{+} \bar{t}) \stackrel{*}{+} \bar{x})=\lambda(\bar{t})$ and $\lambda((\bar{y} \stackrel{*}{+} \bar{t}) \stackrel{*}{+} \bar{y})=\lambda(\bar{t})$, we have

$$
\leqq 4 C \int_{0}^{\delta} \frac{t^{\alpha}}{t} d t+|\bar{f}(\bar{x})-\bar{f}(\bar{y})|\left|\int_{0}^{\delta} D_{n}(t) d t\right| .
$$

Now the function $g(x)=1$ on $(0, \delta),=0$ elsewhere, is a function of bounded variation, and so $S_{n}(0 ; g) \rightarrow g(0)$ by a result of Walsh. But $S_{n}(0 ; g)=\int_{0}^{\delta} D_{n}(t) d t$. Therefore $\left|\int_{0}^{\delta} D_{n}(t) d t\right|$ is bounded, say by $M$. Thus the first integral in (4.8) is

$$
<\frac{4 C \delta^{\alpha}}{\alpha}+M C \delta^{\alpha} \text {. }
$$

Combining (4.9) and (4.11)

$$
\left|\bar{S}_{n}(\bar{x})-\bar{S}_{n}(\bar{y})\right| \leqq C^{*} \delta^{\alpha} \log \frac{1}{\delta}
$$

for small $\delta$ and $\lambda\left({ }_{x}^{*}+\bar{y}\right)<\delta$, the constant $C^{*}$ being independent of $n$, and $\bar{\omega}\left(\delta, \bar{S}_{n}\right)=O\left(\delta^{\alpha} \log 1 / \delta\right)$ uniformly in $n$.

TheOREM IX. (1) A necessary and sufficient condition for $\bar{f}(\bar{x})$ in $L$ to be a Lipschitz $\alpha$ function on $G$ is that $\left\{\bar{\sigma}_{n}(\bar{x})\right\}$, the $(C, 1)$ means of the Fourier development of $\bar{f}(\bar{x})$ relative to the characters of $G$ and Haar measure, be Lipschitz $\alpha$ functions on $G$ uniformly in $n$.

(2) A necessary and sufficient condition for $f(x)$ in L to be a Lipschitz (Wa) function on $[0,1]$ for which $f(x-0)$ exists and is finite at dyadic rationals is that $\left\{\sigma_{n}(x)\right\}$, the $(C, 1)$ means of the W.F.S. of $f(x)$, are Lipschitz $\alpha(W)$ functions uniformly in $n$.

Proof. Suppose $\bar{f}(\bar{x})$ is a Lipschitz $\alpha$ function on $G$. Then 


$$
\left|\bar{\sigma}_{n}(\bar{x})-\bar{\sigma}_{n}(\bar{y})\right|=\left|\int_{G}(\bar{f}(\bar{x} \stackrel{*}{+} \bar{t})-\bar{f}(\bar{y} \stackrel{*}{+} \bar{t})) \bar{K}_{n}(\bar{t}) d \bar{m}\right|
$$

where $\bar{K}_{n}(\bar{t})$ is the $G$-extension of the Fejér kernel. By Theorem I and Lemma II, $\int_{G}\left|\bar{K}_{n}(\bar{t})\right| d \bar{m}=\int_{0}^{1}\left|K_{n}(t)\right| d t \leqq 2$, and hence

$$
\begin{aligned}
\left|\bar{\sigma}_{n}(\bar{x})-\bar{\sigma}_{n}(\bar{y})\right| & \leqq \int_{G}|\bar{f}(\bar{x} \stackrel{*}{+} \bar{t})-\bar{f}(\bar{y} \stackrel{*}{+} \bar{t})|\left|\bar{K}_{n}(\bar{t})\right| d \bar{m} \\
& \leqq C \lambda\left[\left(\bar{x} \stackrel{*}{+} \bar{t} \stackrel{*}{+}^{*}(\bar{y} \stackrel{*}{+} \bar{t})\right]^{\alpha} \int_{G}\left|\bar{K}_{n}(\bar{t})\right| d \bar{m} \leqq 2 C \lambda(\bar{x} \stackrel{*}{+} \bar{y})^{\alpha} .\right.
\end{aligned}
$$

Conversely, suppose $\left\{\bar{\sigma}_{n}(\bar{x})\right\}$ satisfies (4.12) uniformly in $n$. By $(C, 1)$ summability a.e. of the W.F.S. of $f(x)=\bar{f}(\mu(x)), \bar{\sigma}_{n}(\bar{x})$ converges to $\bar{f}(\bar{x})$ a.e. with respect to Haar measure on $G$. Let $\bar{x}_{0}$ be a point of convergence to a finite limit. Then $\left\{\bar{\sigma}_{n}\left(\bar{x}_{0}\right)\right\}$ is a bounded sequence of numbers, say by $M$, and

$$
\left|\bar{\sigma}_{n}(\bar{x})\right|-\left|\bar{\sigma}_{n}\left(\bar{x}_{0}\right)\right| \leqq\left|\bar{\sigma}_{n}(\bar{x})-\bar{\sigma}_{n}\left(\bar{x}_{0}\right)\right| \leqq C \lambda\left(\bar{x} \stackrel{*}{+} \bar{x}_{0}\right)^{\alpha} \leqq C,
$$

and so

$$
\left|\bar{\sigma}_{n}(\bar{x})\right| \leqq M+C
$$

for all $n$ and all $\bar{x}$ in $G$. By Ascoli's theorem, a subsequence $\left\{\bar{\sigma}_{n_{k}}(\bar{x})\right\}$ converges uniformly on $G$ to $\bar{f}(\bar{x})$. Now for any $\bar{x}, \bar{y}$,

$$
|\bar{f}(\bar{x})-\bar{f}(\bar{y})| \leqq\left|\bar{f}(\bar{x})-\bar{\sigma}_{n_{k}}(\bar{x})\right|+\left|\bar{\sigma}_{n_{k}}(\bar{x})-\bar{\sigma}_{n_{k}}(\bar{y})\right|+\left|\bar{\sigma}_{n_{k}}(\bar{y})-\bar{f}(\bar{y})\right| .
$$

Choosing $k$ so large that the first and third terms on the right are each less than

$$
C \lambda(\bar{x}+\bar{y})^{\alpha},
$$

the second term satisfies this by (4.12), and hence $\bar{f}(\bar{x})$ is in Lipschitz class $\alpha$ on $G$, proving the theorem.

5. Theorems of Rogosinski. The following important results of Rogosinski are usually included in a study of the Gibbs phenomenon for Fourier series:

(a) If $\alpha_{n}=O(1 / n)$ and if the series $1 / 2 a_{0}+\sum_{k=1}^{\infty}\left(a_{k} \cos k x+b_{k} \sin k x\right)$ converges to $s(x)$, then $1 / 2\left[S_{n}\left(x+\alpha_{n}\right)+S_{n}\left(x-\alpha_{n}\right)\right] \rightarrow s(x)$.

(b) If this series is summable $(C, 1)$ to $s(x)$, and if $\alpha_{n}=O(1 / n)$, then $\left\{1 / 2\left[S_{n}\left(x+\alpha_{n}\right)+S_{n}\left(x-\alpha_{n}\right)\right]-\left(S_{n}(x)-s(x)\right) \cos n \alpha_{n}\right\} \rightarrow s(x)$. The analogues of these results in the Walsh system will now be investigated.

Theorem X. If $\sum_{k=0}^{\infty} a_{k} \bar{\psi}_{k}(\bar{x})$ is a Walsh series on $G$ which converges to $\bar{s}(\bar{x})$ at $\bar{x}$ in $G$, then for any sequence $\left\{\bar{\alpha}_{n}\right\}$ of $G$ with $\lambda\left(\bar{\alpha}_{n}\right)=O(1 / n)$,

$$
\bar{S}_{n}\left(\bar{x} \stackrel{*}{+} \bar{\alpha}_{n}\right) \rightarrow \bar{s}(\bar{x}) \text { as } n \rightarrow \infty \text {. }
$$


Proof. The following calculation on $[0,1]$ can be done on $G$ for every $\bar{x}$, $\left\{\bar{\alpha}_{n}\right\}, \lambda\left(\bar{\alpha}_{n}\right)=O(1 / n)$.

$$
S_{n}\left(x+\alpha_{n}\right)=\sum_{k=0}^{n-1} a_{k} \psi_{k}(x) \psi_{k}\left(\alpha_{n}\right) \text { for } x \notin E\left(\alpha_{n}\right), \quad\left|E\left(\alpha_{n}\right)\right|=0 .
$$

Using a device found in Fine [1, pp. 391 and 403], let $2^{-\nu-1} \leqq \alpha_{n}<2^{-\nu}$ and write $(n-1)=r 2^{\nu}+s, 0 \leqq s<2^{\nu}$. Then

$$
S_{n}\left(x+\alpha_{n}\right)=\sum_{m=0}^{r-1} \sum_{p=0}^{2^{\nu}-1} a_{2^{\nu}{ }_{m+p}} \psi_{2^{\nu} m+p}(x) \psi_{2^{\nu}{ }_{m+p}}\left(\alpha_{n}\right)+\sum_{p=0}^{g} a_{2^{\nu}{ }_{r+p}} \psi_{2^{\nu}{ }_{r+p}}(x) \psi_{2^{\nu} r+p}\left(\alpha_{n}\right)
$$

for $x \notin E\left(\alpha_{n}\right)$. But is easily verified that $\psi_{2^{\nu} m+p}\left(\alpha_{n}\right)=\psi_{p}\left(\alpha_{n}\right) \psi_{m}\left(2^{v} \alpha_{n}\right)$, and $\psi_{p}\left(\alpha_{n}\right)=1$ when $p<2^{\nu}$. Hence

$$
\begin{aligned}
S_{n}\left(x+\alpha_{n}\right) & =\sum_{m=0}^{r-1} \sum_{p=0}^{2^{\nu}-1} a_{2^{\nu} m+p} \psi_{2^{\nu} m+p}(x) \psi_{m}\left(2^{v} \alpha_{n}\right)+\sum_{p=0}^{\dot{ }} a_{2^{\nu} r+p} \psi_{2^{\nu} r+p}(x) \psi_{r}\left(2^{v} \alpha_{n}\right) \\
& =\sum_{m=0}^{r-1} \psi_{m}\left(2^{v} \alpha_{n}\right)\left[S_{(m+1) 2^{\nu}}(x)-S_{m 2^{\nu}}(x)\right]+\psi_{n-1}\left(\alpha_{n}\right)\left[S_{n}(x)-S_{r 2^{\nu}}(x)\right]
\end{aligned}
$$

where $S_{q}(x)=\sum_{k=0}^{q-1} a_{k} \psi_{k}(x)$ and $\psi_{r}\left(2^{v} \alpha_{n}\right)=\psi_{2^{\nu} r}\left(\alpha_{n}\right)=\psi_{2^{\nu} r}\left(\alpha_{n}\right) \psi_{s}\left(\alpha_{n}\right)=\psi_{n-1}\left(\alpha_{n}\right)$. Or we may write

$$
\begin{aligned}
S_{n}\left(x+\alpha_{n}\right)= & S_{2^{\nu}}(x)+\sum_{m=1}^{r-1} \psi_{m}\left(2^{v} \alpha_{n}\right)\left[S_{(m+1) 2^{\nu}}(x)-S_{m 2^{\nu}}(x)\right] \\
& +\psi_{n-1}\left(\alpha_{n}\right)\left[S_{n}(x)-S_{\mathrm{r} 2^{\nu}}(x)\right]
\end{aligned}
$$

$x \notin E\left(\alpha_{n}\right)$. Now, $\alpha_{n}<2^{c} / n$ for some fixed $c$. Hence $r<n / 2^{v}<2^{c} / 2^{v} \alpha_{n} \leqq 2^{c+1}$ and the number of terms in the right side of (5.1) is bounded. If $S_{n}(x) \rightarrow s(x)$, then the first term on the right side of (5.1) tends also to $s(x)$ and the remaining terms go to zero for all $x \notin \cup_{n=1}^{\infty} E\left(\alpha_{n}\right)$, and the theorem is proved.

Concerning the second result of Rogosinski stated at the beginning of this section, the Walsh analogue might be expected to take the form

$$
\left\{\bar{S}_{n}\left(\bar{x} \stackrel{*}{+} \bar{\alpha}_{n}\right)-\left(\bar{S}_{n}(\bar{x})-\bar{s}(\bar{x})\right) \bar{\psi}_{n-1}\left(\bar{\alpha}_{n}\right)\right\} \rightarrow \bar{s}(\bar{x}) .
$$

This is not a correct result, however. In fact, if $x=\lambda(\bar{x}), \alpha_{n}=\lambda\left(\bar{\alpha}_{n}\right), x+\alpha_{n}$ are not dyadic rationals, (5.2) is equivalent (after a double application of Abel's formula) to

$$
\begin{gathered}
\sum_{k=0}^{n-3}(k+1)\left[\psi_{k}\left(\alpha_{n}\right)-2 \psi_{k+1}\left(\alpha_{n}\right)+\psi_{k+2}\left(\alpha_{n}\right)\right]\left\{\sigma_{k+1}(x)-s(x)\right\} \\
+(n-1)\left[\psi_{n-2}\left(\alpha_{n}\right)-\psi_{n-1}\left(\alpha_{n}\right)\right]\left\{\sigma_{n-1}(x)-s(x)\right\} \\
=\sum_{k=0}^{\infty} b_{n, k}\left\{\sigma_{k+1}(x)-s(x)\right\} \rightarrow 0 .
\end{gathered}
$$


Thus we have a linear transformation of $\left\{\sigma_{k+1}(x)-s(x)\right\}$ by the infinite matrix $\left(b_{n, k}\right)$. Let $(n-1)=2^{\nu}+s, 0 \leqq s<2^{v}$ and choose $x^{\prime}, \alpha_{n}^{\prime}, x^{\prime}+\alpha_{n}^{\prime}$ not dyadic rationals, with $2^{-\nu-1}<\alpha_{n}^{\prime}<2^{-\nu}$. Then $\sum_{k=0}^{\infty}\left|b_{n, k}\right| \geqq n$ and the matrix is not a Toeplitz matrix. Thus there exists a sequence $\left\{\sigma_{k}^{\prime}\left(x^{\prime}\right)\right\}$ converging to $s^{\prime}\left(x^{\prime}\right)$ but for which (5.2) does not hold for $\left\{\alpha_{n}^{\prime}\right\}$. Moreover, it is possible to find a Walsh series $\sum_{k=0}^{\infty} a_{k} \psi_{k}(x)$ which at $x^{\prime}$ has the $(C, 1)$ means $\left\{\sigma_{k}^{\prime}\left(x^{\prime}\right)\right\}$.

Theorem XI. Let $f(x) \in L^{k}(0,1), 1<k<\infty$, and $\left\{n_{k}\right\}$ be a lacunary sequence of integers $n_{k+1} / n_{k}>q>1$. Then for any sequence $0 \leqq \alpha_{n}=O(1 / n)$, the W.F.S. of $f(x)$ satisfies $S_{n_{k}}\left(x+\alpha_{n_{k}}\right) \rightarrow f(x)$ a.e. as $k \rightarrow \infty$.

Proof. Using (5.1) with $n=n_{k}, 2^{-\nu-1} \leqq \alpha_{n_{k}}<2^{-\nu}$, and $n_{k}-1=r 2^{\nu}+p$, $0 \leqq p<2^{v}$ it is apparent that the partial sums on the right are lacunary if $n=n_{k}$ and, as before, there is a finite number of terms. By the following lemma due to Paley [9] the right side tends to $f(x)$ a.e. and the result is proved.

Lemma III. If $f(x) \in L^{k}(0,1), 1<k<\infty$, and $\left\{n_{k}\right\}$ is a lacunary sequence of integers $n_{k+1} / n_{k}>q>1$, the partial sums of the W.F.S. of $f(x)$ satisfy $S_{n_{k}}(x)$ $\rightarrow f(x)$ a.e.

The next theorem resembles the second theorem of Rogosinski.

Theorem XII. If $f(x) \in L^{k}(0,1), 1<k<\infty$, and $\left\{\alpha_{n}\right\}$ is any sequence satisfying $0 \leqq \alpha_{n}=O(1 / n)$, then the partial sums of the W.F.S. of $f(x)$ satisfy

$$
\left[S_{n}\left(x+\alpha_{n}\right)-\left(S_{n}(x)-f(x)\right) \psi_{n-1}\left(\alpha_{n}\right)\right] \rightarrow f(x) \text { a.e. }
$$

Proof. Formula (5.1) may be rewritten as

$$
\begin{array}{r}
S_{n}\left(x+\alpha_{n}\right)-\psi_{n-1}\left(\alpha_{n}\right)\left[S_{n}(x)-f(x)\right] \\
=S_{2^{\nu}}(x)+\sum_{m=1}^{r-1} \psi_{m}\left(2^{\nu} \alpha_{n}\right)\left[S_{(m+1) 2_{\nu}}^{(x)}-S_{m 2^{\nu}}(x)\right]+\psi_{n-1}\left(\alpha_{n}\right)\left[f(x)-S_{r 2^{\nu}}(x)\right] \\
\text { for } x \notin E\left(\alpha_{n}\right) .
\end{array}
$$

The relation holds for each $n$ if $x \notin \cup_{n-1}^{\infty} E\left(\alpha_{n}\right)$. As $n \rightarrow \infty, \nu \rightarrow \infty$, and the collections $\left\{S_{m 2}{ }(x)\right\}, m=1,2, \cdots, r \leqq 2^{c+1}$ are each lacunary sequences of partial sums. By Lemma III each such sequence tends to $f(x)$ a.e. so the right side converges a.e. to $f(x)$.

This section will end with the proof of a valuable formula for any integrable function $f(x)$ in terms of the partial sums of its W.F.S. This result (which is an analogue of a well known result for the trigonometrical system, e.g., see Marcinkiewicz and Zygmund [7, pp. 3, 4]) is not related to the theorems of Rogosinski, but depends on some of the facts used in the proofs of these theorems, and so it is included in this section.

TheOREM XIII. If $f(x) \in L(0,1)$ and if the W.F.S. of $f(x)$ has partial sums 
satisfying $\lim \sup _{n-\infty} S_{n}(x)<+\infty$ for $x \in E$ where $E$ is any set of positive measure, then for almost every $x \in E$ we have

$$
\liminf _{n \rightarrow \infty} S_{n}(x)>-\infty
$$

and

$$
f(x)=\frac{1}{2}\left\{\liminf _{n \rightarrow \infty} S_{n}(x)+\limsup _{n \rightarrow \infty} S_{n}(x)\right\} .
$$

Proof. The function $S^{*}(x)=\lim \sup _{n-\infty} S_{n}(x)<+\infty$ is a measurable function on $E$, and following Lusin, there exists a set $F \subset E$ such that the complement of $F$ relative to $E$ has measure less than $\epsilon / 2$, and $S^{*}(x)$ is continuous on $F$ relative to $F$.

Also the sequence of functions $\left\{\right.$ 1.u.b. $\left.{ }_{k \geq n} S_{k}(x)\right\}$ converges on $F$ to $S^{*}(x)$, and therefore there is a measurable subset $E^{\prime}$ of $F$ such that $\left|E^{\prime}-F\right|<\epsilon / 2$, and the sequence converges uniformly on $E^{\prime}$.

Let $x$ be a point of metric density of $E^{\prime}$. Then the relative density of any interval containing $x$ and of sufficiently small diameter is close to unity. More specifically, if $\alpha_{m}(x), \beta_{m}(x)$ are defined by

$$
\alpha_{m}(x)=\frac{p}{2^{m}} \leqq x<\frac{p+1}{2^{m}}=\beta_{m}(x),
$$

then there exists $\nu_{0}$ such that for $\nu \geqq \nu_{0}$

$$
\left|E^{\prime} \cap\left(\alpha_{\nu}(x), \beta_{\nu}(x)\right)\right|>\frac{1}{2} \cdot 2^{-\nu}
$$

For every $n$ such that $(n-1)>2^{\nu 0}$, define $\nu$ by $2^{\nu} \leqq(n-1)<2^{\nu+1}$, and consider the image of the interval $I: 2^{-(\nu+1)}<\alpha<2^{-\nu}$ under transformation $T_{x}(I)$ $=\{x \dot{+} \alpha, \alpha \in I\}$. We have $\left|T_{x}(I)\right|=|I|=2^{-(\nu+1)}$. However, except for a set of measure zero $T_{x}(I) \subset\left(\alpha_{\nu}(x), \beta_{\nu}(x)\right)$, and so $E \cap T_{x}(I)$ is not void, i.e., there exists an $\alpha=\alpha_{n}$ such that $\left(x \dot{+} \alpha_{n}\right) \in E^{\prime}$ and $\alpha_{n} \in I$. For such $\alpha_{n}$ by (5.1) with $r=1$, as is now the case,

$$
S_{n}\left(x+\alpha_{n}\right)=S_{2^{v}}(x)\left[1-\psi_{n-1}\left(\alpha_{n}\right)\right]+\psi_{n-1}\left(\alpha_{n}\right) S_{n}(x) .
$$

But $\psi_{n-1}\left(\alpha_{n}\right)=\psi_{2} v\left(\alpha_{n}\right)=\psi_{1}\left(2^{v} \alpha_{n}\right)=-1$ since $\alpha_{n} \in I$. Thus

$$
S_{n}\left(x+\alpha_{n}\right)=2 S_{2}(x)-S_{n}(x) \text { for each } n \text {. }
$$

Now in view of the continuity of $S^{*}(x)$ over $E^{\prime}$ relative to $E^{\prime}$ and the fact that $x$ is in $E^{\prime}$ and for each $n,\left(x+\alpha_{n}\right)$ belongs to $E^{\prime}$, we have

$$
S_{n}\left(x+\alpha_{n}\right) \leqq S^{*}\left(x+\alpha_{n}\right)+\epsilon_{n} \leqq S^{*}(x)+o(1), n \text { large. }
$$

By (5.4)

$$
2 S_{2}(x) \leqq S^{*}(x)+o(1)+S_{n}(x), \text { large } n .
$$


But $S_{2} r(x)=f(x)+\epsilon_{n}(x), \epsilon_{n}(x) \rightarrow 0$ a.e. Assume that the $x$ under consideration is such a point of convergence and $f(x)$ is finite (which it is a.e.). Then $S^{*}(x)+o(1) \geqq-S_{n}(x)+2 f(x)+2 \epsilon_{n}(x)$, and hence $S^{*}(x)-2 f(x) \geqq-S_{*}(x)$ $=-\lim \inf _{n \rightarrow \infty} S_{n}(x)$. Consequently $\lim \inf _{n \rightarrow \infty} S_{n}(x)>-\infty$, and the first assertion of the theorem is proved since $E^{\prime}$ is of measure arbitrarily close to that of $E$.

Knowing $S_{*}(x)>-\infty$ we may write

$$
f(x) \leqq \frac{1}{2}\left\{S^{*}(x)+S_{*}(x)\right\} \text { a.e. on } E .
$$

Suppose now that the argument were repeated starting with the inequality $S_{*}(x)>-\infty$ as the given condition. All inequalities would be reversed, and from this would follow $1 / 2\left\{S^{*}(x)+S_{*}(x)\right\} \leqq f(x)$ a.e. on $E$. Combining this with (5.5) proves the theorem.

6. Transformations of Walsh series. In the theory of trigonometric series a number of results have been established (see Zygmund [21]) which identify Fourier series with classes of functions. A theory of transformation of series from one class to another class by means of multipliers has also been established. In this section, the Walsh analogues are obtained. If a Walsh series $\sum_{k=0}^{\infty} a_{k} \psi_{k}(x)$ is the Fourier series of $f(x)$ which belongs to a class of functions $\Omega$, then we shall say (following the classical exposition) that the series itself belongs to $\Omega$. For any numerical sequence $\left\{\lambda_{k}\right\}$ consider besides the Walsh series the two series

$$
\sum_{k=0}^{\infty} \lambda_{k} \psi_{k}(x)
$$

and

$$
\sum_{k=0}^{\infty} \lambda_{k} a_{k} \psi_{k}(x)
$$

Given two classes $P, Q$, of Walsh series, the class of sequences $\left\{\lambda_{k}\right\}$ denoted by $(P, Q)$ is composed of sequences which transform $P$ into $Q$, that is, whenever the given Walsh series belongs to $P,(6.2)$ belongs to $Q$. We first identify the series belonging to certain classes of functions.

TheOREM XIV. A necessary and sufficient condition that a Walsh series should belong to the class $B$ of essentially bounded periodic functions on $[0,1]$, is the existence of a constant $M$ such that the $(C, 1)$ means of the series satisfies $\left|\sigma_{k}(x)\right| \leqq 2 M$, all $k$, all $x$.

Proof. If $|f(x)| \leqq M$ a.e., then by Lemma II $\left|\sigma_{k}(x)\right| \leqq \int_{0}^{1}|f(x+t)|\left|K_{k}(t)\right| d t$ $\leqq 2 M$, all $x$ and $k$.

If $\left|\sigma_{k}(x)\right| \leqq 2 M$, all $k$ and all $x$, then 


$$
4 M^{2} \geqq \int_{0}^{1} \sigma_{k}^{2}(x) d x=\sum_{j=0}^{k-1} a_{j}^{2}\left(1-\frac{j}{k}\right)^{2} \geqq \sum_{j=0}^{\nu} a_{j}^{2}\left(1-\frac{j}{k}\right)^{2}
$$

where $\nu$ is any fixed integer less than $(k-1)$. But as $k \rightarrow \infty$ this implies $\sum_{j=0}^{y} a_{j}^{2} \leqq 4 M^{2}$, and since $\nu$ is arbitrary, $\sum_{j=0}^{\infty} a_{j}^{2}<+\infty$. There exists an $f(x)$ in $L^{2}$ such that $f(x) \sim \sum_{j=0}^{\infty} a_{j} \psi_{j}(x)$. By the result of Paley [9], $\sigma_{k}(x) \rightarrow f(x)$ a.e. and since $\left|\sigma_{k}(x)\right| \leqq 2 M$ for all $k$ and all $x,|f(x)| \leqq 2 M$ a.e.

In characterizing series which belong to the class of continuous functions, the continuity on the group $G$ is required. The usual characterization (uniform convergence of the $(C, 1)$ means $\left.\sigma_{k}(x)\right)$ fails because the Walsh $(C, 1)$ means are only continuous $(W)$. For example, if $f(x)$ is a Walsh polynomial, $\sigma_{k}(x ; f) \rightarrow f(x)$ uniformly, but $f(x)$ is not continuous.

Theorem XV. A necessary and sufficient condition for a Walsh series on $G$ to belong to the class $C$ of continuous functions on $G$ is that the $(C, 1)$ means $\left\{\bar{\sigma}_{k}(\bar{x})\right\}$ of the series should converge uniformly.

Proof. If $\sum_{k=0}^{\infty} a_{k} \bar{\psi}_{k}(\bar{x})$ is the Fourier series of a function $\bar{f}(\bar{x})$ continuous on $G$, then $\left\{\bar{\sigma}_{k}(\bar{x})\right\}$ converges uniformly. This is the theorem of Fejerr on the group $G$.

On the other hand, if $\left\{\bar{\sigma}_{k}(\bar{x})\right\}$ converges uniformly, define $\bar{f}(\bar{x})$ $=\lim _{k \rightarrow \infty} \bar{\sigma}_{k}(\bar{x})$. Note that $\bar{f}(\bar{x})$ is continuous. Consider

$$
\left\{j \text { th coefficient of } \bar{\sigma}_{k}(\bar{x})\right\}=\left(1-\frac{j}{k}\right) a_{j}=\int_{G} \bar{\sigma}_{k}(\bar{x}) \psi_{j}(\bar{x}) d \bar{m} \text {. }
$$

Now as $k \rightarrow \infty$ the left side converges to $a_{j}$ while the right side converges to $\int_{G} \bar{f}(\bar{x}) \psi_{j}(\bar{x}) d \bar{m}$.

In the classical theory, the concept of a Fourier-Stieltjes series is introduced. To every function $F(x)$ of bounded variation on $[0,2 \pi]$ corresponds a trigonometrical series whose coefficients are Riemann-Stieltjes integrals, e.g., $(1 / \pi) \int_{0}^{2 \pi} \cos k t d F(t)$.

In the Walsh case the corresponding integrals $\int_{0}^{1} \psi_{k}(t) d F(t)$ need not exist as Riemann-Stieltjes integrals. This is the case, for example, when the function of bounded variation $F(x)$ has a common discontinuity with $\psi_{k}(x)$. It is necessary, therefore, either to consider the problem in the context of the group $G$ where $\bar{\psi}_{k}(\bar{x})$ is continuous or to generalize the Stieltjes integral involved and/or restrict $F(t)$. The first approach leads to the difficulty of defining functions of bounded variation on the unordered group $G$. The LebesgueStieltjes integral or modified Pollard-Moore Stieltjes integral of Hildebrandt [5] are generalizations for which $\int_{0}^{1} \psi_{k}(t) d F(t)$ does exist for every $F(t)$ of bounded variation on $[0,1]$, however, this approach has thus far involved other difficulties. The validity of the sufficiency portion of the classical necessary and sufficient condition for a series to be a Fourier-Stieltjes series 
$\int_{0}^{1}\left|\sigma_{n}(x)\right| d x \leqq V$ has thus far been neither proved nor disproved by the author. We therefore define $S^{\prime}$ as the class of series $\sum_{k=0}^{\infty} a_{k} \psi_{k}(x)$ whose coefficients are $a_{k}=\int_{0}^{1} \psi_{k}(t) d F(t)$ where $F(t)$ is continuous and of bounded variation.

THEOREM XVI. If $\sum_{k=0}^{\infty} a_{k} \psi_{k}(x)$ belongs to $S^{\prime}, \int_{0}^{1}\left|\sigma_{n}(x)\right| d x \leqq 2 V$ and $1 / n \sum_{k=0}^{n-1} a_{k} \psi_{k}(x)$ converges to zero uniformly in $x$ and conversely.

Proof. Except when $(x+t)$ is a dyadic rational (the set of such $t$ for fixed $x$ has $F$-measure zero)

$$
K_{n}(x+t)=K_{n}(x, t)=\sum_{k=1}^{n} \sum_{r=0}^{k-1} \psi_{r}(x) \psi_{r}(t) / n
$$

and hence $\left|\sigma_{n}(x)\right| \leqq \int_{0}^{1}\left|K_{n}(x \dot{+} t)\right||d F(t)|$. Then with $(n-1)=2^{\prime}+n^{\prime}$, $0 \leqq n^{\prime}<2^{\nu}$,

$$
\begin{aligned}
\int_{0}^{1}\left|\sigma_{n}(x)\right| d x & \leqq \int_{0}^{1}\left(\int_{0}^{1}\left|K_{n}(x+t)\right||d F(t)|\right) d x \\
& =\int_{0}^{1}\left(\sum_{i=0}^{2^{\nu+1}-1}\left|K_{n}\left(x+\frac{i}{2^{\nu+1}}\right)\right| \int_{i / 2^{\nu+1}}^{(i+1) / 2^{\nu+1}}|d F(t)|\right) d x \leqq 2 V
\end{aligned}
$$

since $K_{n}(x+t)$ is constant (for fixed $x$ ) for $t$ in $\left[i / 2^{v+1},(i+1) / 2^{v+1}\right.$ ), and $\int_{0}^{1}\left|K_{n}(u)\right| d u \leqq 2$ by Lemma II.

Moreover, let $n=\sum_{i=0}^{\nu} x_{i} 2^{i}, x_{i}=0,1, x_{\nu}=1$, and let $n_{j}=\sum_{i=0}^{j} x_{i} 2^{i}$, $0 \leqq j \leqq \nu$. Repeating the formula $D_{n}(x)=D_{2^{\nu}}(x)+\psi_{2^{\nu}}(x) D_{n-2^{\nu}}(x) k$ times $(k<\nu)$ yields (using 5.3)

$$
\begin{aligned}
\frac{1}{n}\left|\sum_{r=0}^{n-1} a_{r} \psi_{r}(x)\right| \leqq & \sum_{i=0}^{k-1} \frac{x_{\nu-i}}{n}\left|\int_{0}^{1} \psi_{n-n_{\nu-i}}(x+t) D_{2^{\nu-i}}(x+t) d F(t)\right| \\
& +\frac{1}{n}\left|\int_{0}^{1} \psi_{n-n_{\nu-k}}(x+t) D_{n_{\nu-k}}(x+t) d F(t)\right| D_{0}(u) \equiv 0 . \\
\leqq & \sum_{i=0}^{k-1} \frac{x_{\nu-i} 2^{\nu-i}}{n}\left[V\left(\beta_{\nu-i}(x)\right)-V\left(\alpha_{\nu-i}(x)\right)\right] \\
& +\frac{2^{\nu-k+1}[V(1)-V(0)]}{n} \\
\leqq & {\left[V\left(\beta_{\nu-k+1}(x)\right)-V\left(\alpha_{\nu-k+1}(x)\right)\right]+\frac{1}{2^{k-1}}[V(1)-V(0)] . }
\end{aligned}
$$

The first term can be made small uniformly in $x$ by choosing $n$ large and the second small by choosing $k$ large.

Conversely, if $\int_{0}^{1}\left|\sigma_{n}(x)\right| d x \leqq 2 V$ and $1 / n \sum_{k=0}^{n-1} a_{k} \psi_{k}(x) \rightarrow 0$ uniformly in $x$, define $F_{n}(x)=\int_{0}^{x} \sigma_{n}(t) d t$. Then (cf. Zygmund [21, p. 80]) there exists $F_{n_{k}}(x)$, a uniformly bounded subsequence converging everywhere to $F(x)$ of bounded variation. 
For each $k\left(n_{k}>i\right)$

$$
\left(1-\frac{i}{n_{k}}\right) a_{\imath}=\int_{0}^{1} \psi_{i}(x) \sigma_{n_{k}}(x) d x=\int_{0}^{1} \psi_{i}(x) d F_{n_{k}}(x) .
$$

With $i=2^{s_{i}}+i^{\prime}, 0 \leqq i^{\prime}<2^{s_{i}}$, let $I_{j}=\left[j / 2^{s_{i+1}},(j+1) / 2^{s_{i+1}}\right), j=0,1,2, \cdots$, $\left(2^{s_{i+1}}-1\right)$ be the intervals of constancy of $\psi_{i}(x)$. Then

$$
\left(1-\frac{i}{n_{k}}\right) a_{i}=\sum_{j=0}^{\left(2^{s_{i}+1}-1\right)} \psi_{i}\left(I_{j}\right)\left[F_{n_{k}}\left(\frac{j+1}{2^{s_{i}+1}}\right)-F_{n_{k}}\left(\frac{j}{2^{s^{i}+1}}\right)\right] .
$$

Letting $k \rightarrow \infty$, and replacing each $F_{n_{k}}(x)$ by $F(x)$ we have $a_{i}=\int_{0}^{1} \psi_{i}(x) d F(x)$ if $F(x)$ is continuous.

An idea of Fine $[1$, p. 406] may be used to demonstrate the continuity of $F(x) . F(x-0)$ and $F(x+0)$ exist everywhere and hence it is enough to find dyadic rational sequences $\left\{r_{j}^{+}\right\}$and $\left\{r_{j}^{-}\right\}$converging to $x$ from right and left respectively and $F\left(r_{j}^{+}\right) \rightarrow F(x), F\left(r_{j}^{-}\right) \rightarrow F(x)$.

By definition $F_{n_{k}}(x)=\sum_{i=0}^{\left(n_{k}-1\right)}\left(1-i / n_{k}\right) a_{i} J_{i}(x)$, where $J_{i}(x)=\int_{0}^{x} \psi_{i}(t) d t$. For $i$ large enough $J_{i}\left(\beta_{n}(x)\right)=J_{i}\left(\alpha_{n}(x)\right)=0$, hence

$$
\begin{aligned}
F\left(\beta_{n}(x)\right)-F\left(\alpha_{n}(x)\right) & =\lim _{x \rightarrow \infty} \sum_{i=0}^{\left(2^{n}-1\right)}\left(1-\frac{i}{n_{k}}\right) a_{i}\left[J_{i}\left(\beta_{n}(x)-J_{n}\left(\alpha_{n}(x)\right)\right]\right. \\
& =\lim _{x \rightarrow \infty} \sum_{i=0}^{\left(2^{n}-1\right)}\left(1-\frac{i}{n_{k}}\right) a_{i} \int_{\alpha_{n}(x)}^{\beta_{n}(x)} \psi_{i}(t) d t \\
& =\int_{\alpha_{n}(x)}^{\beta_{n}(x)} S_{2^{n}}(t) d t=\frac{S_{2^{n}}(x)}{2^{n}} \rightarrow 0
\end{aligned}
$$

by assumption. Hence if $x$ is not a dyadic rational, we identify $\left\{\beta_{n}(x)\right\}$ with $\left\{r_{j}^{+}\right\},\left\{\alpha_{n}(x)\right\}$ with $\left\{r_{j}^{-}\right\}$. If $x$ is a dyadic rational, $\alpha_{n}(x)=x$ for large $n$, and hence while $\left\{\beta_{n}(x)\right\}$ is still $\left\{r_{j}^{+}\right\}$, we define $\left\{r_{j}^{-}\right\} \equiv\left\{\alpha_{n}^{\prime}(x)\right\}=\left\{\alpha_{n}(x)-1 / 2^{n}\right\}$. Then $F(x)-F\left(\alpha_{n}^{\prime}(x)\right)=S_{2^{n}}\left(\alpha_{n}^{\prime}\right) / 2^{n} \rightarrow 0$ since by assumption $S_{2^{n}}(u) / 2^{n} \rightarrow 0$ uniformly in $u$ and so the theorem is proved.

THEOREM XVII. A necessary and sufficient condition that $\sum_{k=0}^{\infty} a_{k} \Psi_{k}(\bar{x})$ be the Walsh-Fourier series of a function $\bar{f}(\bar{x}) \in L(G)$ is that the $(C, 1)$ means satisfy $\int_{G}\left|\bar{\sigma}_{m}(\bar{x})-\bar{\sigma}_{n}(\bar{x})\right| d \bar{m} \rightarrow 0$, as $m, n \rightarrow \infty$.

Proof. The necessity is immediate from Theorem VI. It is enough to prove the sufficiency on the unit interval. By completeness of $L(0,1)$ there exists $f(x) \in L(0,1)$ such that $\int_{0}^{1}\left|f(x)-\sigma_{n}(x)\right| d x \rightarrow 0$ as $n \rightarrow \infty$. Define $F_{n}(x)$ $=\int_{0}^{x} \sigma_{n}(t) d t, F(x)=\int_{0}^{x} f(t) d t$. Then

$$
\left|F(x)-F_{n}(x)\right| \leqq \int_{0}^{1}\left|f(t)-\sigma_{n}(t)\right| d t \rightarrow 0 \text { as } n \rightarrow \infty,
$$

and so $F_{n}(x) \rightarrow F(x)$. 
As in (6.3), if $n>k,(1-k / n) a_{k}=\int_{0}^{1} \sigma_{n}(x) \psi_{k}(x) d x=\int_{0}^{1} \psi_{k}(x) d F_{n}(x)$. Arguing as in (6.4), it is clear that as $n \rightarrow \infty, a_{k}=\int_{0}^{1} \psi_{k}(x) d F(x)=\int_{0}^{1} \psi_{k}(x) f(x) d x$.

The proofs of the next results parallel closely the proofs of the classical analogues.

Theorem XVIII. If $\sum_{k=0}^{\infty} \lambda_{k} \psi_{k}(x)$ belongs to $S^{\prime}$, then $\left\{\lambda_{n}\right\}$ belongs to $(B, B),(C, C(W))$, and $(L, L)$. Conversely if $\left\{\lambda_{n}\right\}$ belongs to any of these classes and if also $\sum_{k=0}^{n-1} \lambda_{k} \psi_{k}(x) / n \rightarrow 0$ uniformly in $x$, then $\sum_{k=0}^{\infty} \lambda_{k} \psi_{k}(x)$ belongs to $S^{\prime}$ (cf. Zygmund [21]).

Proof. Let $\sigma_{n}(x), q_{n}(x)$, and $\sigma_{n}^{*}(x)$ denote respectively the $(C, 1)$ means of the series $\sum_{k=0}^{\infty} a_{k} \psi_{k}(x), \sum_{k=0}^{\infty} \lambda_{k} \psi_{k}(x)$, and $\sum_{k=0}^{\infty} a_{k} \lambda_{k} \psi_{k}(x)$. Assuming the first series to belong to $B$ and the second series to belong to $S^{\prime}$, we have

$$
\sigma_{n}^{*}(x)=\sum_{k=0}^{n-1}\left(1-\frac{k}{n}\right)\left(\int_{0}^{1} \psi_{k}(t) d F(t)\right) a_{k} \psi_{k}(x)=\int_{0}^{1} \sigma_{n}(x+t) d F(t) .
$$

By Theorem XIV, $\left|\sigma_{n}(x \dot{+} t)\right| \leqq M$, and hence $\left|\sigma_{n}^{*}\right| \leqq M V$. By Theorem XIV $\sum_{k=0}^{\infty} a_{k} \lambda_{k} \psi_{k}(x)$ belongs to $B$.

If the first series belongs to $C$, then $\left[\sigma_{n}(x)-\sigma_{m}(x)\right] \rightarrow 0$ uniformly for $x \in[0,1]$ by Fejér's theorem. But then

$$
\left|\sigma_{n}^{*}(x)-\sigma_{m}^{*}(x)\right| \leqq \int_{0}^{1}\left|\sigma_{n}(x+t)-\sigma_{m}(x+t)\right||d F(t)| \rightarrow 0
$$

uniformly. This implies that $\left\{\bar{\sigma}_{n}^{*}(\bar{x})\right\}$ converges uniformly on $G$ and hence by Theorems XV and IV the transformed series is in $C(W)$.

If the given series is in $L, \int_{0}^{1}\left|\sigma_{n}(x)-\sigma_{m}(x)\right| d x \rightarrow 0$ by Theorem VI. Hence from (6.6)

$$
\begin{aligned}
\int_{0}^{1}\left|\sigma_{n}^{*}(x)-\sigma_{m}^{*}(x)\right| d x & \leqq \int_{0}^{1} \int_{0}^{1}\left|\sigma_{n}(x+t)-\sigma_{m}(x+t)\right||d F(t)| d x \\
& \leqq V \int_{0}^{1}\left|\sigma_{n}(x)-\sigma_{m}(x)\right| d x \rightarrow 0 .
\end{aligned}
$$

By Theorem XVII, the transformed series belongs to $L$.

The proof of the necessity portion of the theorem proceeds exactly as in the classical case and will not be given (cf. Zygmund [21, pp. 101-102]).

TheOREM XIX. If $\sum_{k=0}^{\infty} \lambda_{k} \psi_{k}(x)$ belongs to $L,\left\{\lambda_{n}\right\}$ belongs to $\left(S^{\prime}, L\right)$ and $(B, C(W))$.

Proof. Given $\sum_{k=0}^{\infty} a_{k} \psi_{k}(x)$ in $S^{\prime}$, it is clear that

$$
\begin{aligned}
\int_{0}^{1}\left|\sigma_{n}^{*}(x)-\sigma_{m}^{*}(x)\right| d x & \leqq \int_{0}^{1} \int_{0}^{1}\left|q_{n}(x+t)-q_{m}(x+t)\right||d F(t)| d x \\
& \leqq V \int_{0}^{1}\left|q_{n}(x)-q_{m}(x)\right| d x \rightarrow 0
\end{aligned}
$$


since $\sum_{k=0}^{\infty} \lambda_{k} \psi_{k}(x)$ belongs to $L$. But then by Theorems I and XVII, the series $\sum_{k=0}^{\infty} a_{k} \lambda_{k} \psi_{k}(x)$ is in $L$.

If $\sum_{k=0}^{\infty} a_{k} \psi_{k}(x)$ belongs to $B$,

$$
\left|\sigma_{n}^{*}(x)-\sigma_{m}^{*}(x)\right| \leqq \int_{0}^{1}\left|q_{n}(t)-q_{m}(t)\right||f(x+t)| d t \leqq M \int_{0}^{1}\left|q_{n}(t)-q_{m}(t)\right| d t
$$

where $M$ is the essential upper bound of $f$. Since $\sum_{k=0}^{\infty} \lambda_{k} \psi_{k}(x)$ is in $L$, the right side tends to zero and so $\left\{\sigma_{n}^{*}(x)\right\}$ is a uniformly convergent sequence. An application of Theorems XV and IV shows $\sum_{k=0}^{\infty} a_{k} \lambda_{k} \psi_{k}(x)$ to be in $C(W)$.

7. Lacunary Walsh Series. In this section, analogues are developed for a number of interesting results from the theory of lacunary trigonometric series. The first is for a result of Zygmund [20], the proof differing from that of Zygmund's only in respect to certain details which are peculiar to the Walsh functions. In general, the results of this section may be restated on the group $G$.

THEOREM XX. If $\sum_{k=0}^{\infty} a_{k} \psi_{n_{k}}(x)$ is a lacunary Walsh series whose partial sums $S_{n}(x)$ oscillate finitely at each point of an interval $I \subset[0,1]$, then $\sum_{k=0}^{\infty}\left|a_{k}\right|<\infty$.

Proof. We begin by proving the lemma of Baire for the Walsh series.

Lemma IV. If the partial sums of a Walsh series oscillate finitely at each point of an interval $I \subset[0,1]$, there exists a constant $M$ and subinterval $[a, b] \subset I$ such that $\left|S_{n}(x)\right| \leqq M$ for all $n$ and all $x \in[a, b]$.

Proof. Let $I^{\prime}$ be a closed dyadic subinterval of $I$ and consider the neighborhood $N$ of $G$ which is mapped onto $I^{\prime}$ under $\lambda$. Define $E_{i k}=\left\{\bar{x} \in N|| \bar{S}_{i}(\bar{x}) \mid\right.$ $\leqq k\}$. Each $E_{i k}$ is a closed set in $G$ since $\bar{S}_{i}(\bar{x})$ is continuous on $G$. Then $\bigcap_{i=1}^{\infty} E_{i k}=E_{k}$ is a closed set, and $N^{\prime}=\bigcup_{k=1}^{\infty} E_{k}$ is a union of closed sets. The set $N^{\prime}$ contains every $\bar{x} \in N$ which does not end in a sequence of 1's. This follows from the fact that for all such $\bar{x}$ there is an $x$ in $I^{\prime}$ for which $\mu(x)=\bar{x}$ and $\bar{S}_{i}(\bar{x})=\bar{S}_{i}(\mu(x))=S_{i}(x)$ by (1.8). But then $\left\{S_{i}(x)\right\}$ is a finitely oscillating sequence, and so $\bar{x}$ belongs to $E_{i k}$ for every $i$ when $k$ is sufficiently large.

This implies $N=\left(\bigcup_{k=1}^{\infty} E_{k}\right) \cup Z$ where $Z$ is at most a denumerable set. But $N$ is a neighborhood of $G$ and is not of the first category and hence one of the $E_{k}$ is not nowhere dense. Call this set $E_{M}$. Then $E_{M}$ contains a neighborhood $\bar{N}$ of $G$.

By definition of $E_{M},\left|\bar{S}_{i}(\bar{x})\right| \leqq M$ for all $i$ and all $\bar{x}$ in $\bar{N}$. But then $\left|S_{i}(x)\right|$ $\leqq M$ on $[a, b]$, a dyadic subinterval of $I^{\prime}$ which is part of the image of $\bar{N}$ under $\lambda$.

Suppose now that $n_{k+1} / n_{k}$ exceeds $q>3$ and set $P_{N}(x)=\prod_{k=1}^{N}\left(1+\epsilon_{k} \psi_{n_{k}}(x)\right)$, $\epsilon_{k}= \pm 1$. Then

$$
\int_{a}^{b} P_{N}(x) d x=\int_{a}^{b}\left(1+\sum_{\nu} \alpha_{\nu} \psi_{\nu}(x)\right) d x
$$


when expanded because products of $\psi$ 's combine according to the following combinatorial law (Fine [2]): if for any integers $m, n$ we write $m=\sum_{i=0}^{\infty} x_{i} 2^{i}$, $n=\sum_{i=0}^{\infty} y_{i} 2^{i}, x_{i}, y_{i}=0,1$, and we define

(7.2) $\quad m \oplus n=\sum_{i=0}^{\infty}\left|x_{i}-y_{i}\right| 2^{i}$, then $\psi_{m}(x) \psi_{n}(x)=\psi_{m} \oplus_{n}(x)$ for all $x$.

Let $G_{h k}$ be the sum of all terms in the expansion of $P_{N}(x)$ which consist of a product of $h \psi$ 's and wherein $n_{k}$ is the largest of the subscripts in such a product. Then

$$
\begin{aligned}
& \left|\int_{a}^{b}\left(G_{1 k}+G_{2 k}+\cdots+G_{k k}\right) d x\right| \\
& \leqq \sum_{h=1}^{k}\left(\begin{array}{c}
\text { number of terms } \\
\text { in } G_{h k}
\end{array}\right)\left(\max _{\psi_{\nu} \in G_{h k}}\left|\int_{a}^{b} \psi_{\nu}(x) d x\right|\right) .
\end{aligned}
$$

Now since $n_{k+1} / n_{k}>q>3$, each integer $n_{k}$ necessarily contains a higher power of 2 in its dyadic expansion than its predecessor had. It follows that no "sum" $\nu=n_{k_{i_{1}}} \oplus n_{k_{i_{2}}} \oplus \cdots \oplus n_{k_{i_{j}}}, n_{k_{i_{1}}}<n_{k_{i_{2}}}<\cdots<n_{k_{i_{j}}}$ can be zero. Hence

$$
\begin{aligned}
\left|\int_{a}^{b}\left(P_{N}(x)-1\right) d x\right| & \leqq \sum_{k=1}^{\infty}\left|\int_{a}^{b}\left(G_{1 k}+G_{2 k}+\cdots+G_{k k}\right) d x\right| \\
& \leqq \sum_{k=1}^{\infty} \sum_{h=1}^{k}\left(\begin{array}{c}
k-1 \\
h-1
\end{array}\right)\left(\max _{\nu \text { appearing in } G_{k k}}\left|\int_{a}^{b} \psi_{\nu}(x) d x\right|\right) .
\end{aligned}
$$

Now if the "sum" $\nu$ belongs to $G_{h k}$, its greatest "summand" is $n_{k}=2^{8 n_{k}}$ $+n_{k}^{\prime}, 0 \leqq n_{k}^{\prime}<2^{s n_{k}}$. Thus

$$
\begin{aligned}
\left|\int_{a}^{b} \psi_{\nu}(x) d x\right| & =\left|J_{\nu}(b)-J_{\nu}(a)\right|=\left|\psi_{\nu}(b)\left(b-\gamma_{s_{\boldsymbol{n}_{k}}}(b)\right)-\psi_{\nu}(a)\left(a-\gamma_{s_{n_{k}}}(a)\right)\right| \\
& \leqq \frac{4}{n_{k}}
\end{aligned}
$$

where $\gamma_{s_{n_{k}}}(b)$ is defined as that one of $\alpha_{s_{n_{k}}}(b), \beta_{s_{n_{k}}}(b)$ (defined in (5.3)) which is closer to $b$ and similarly for $\gamma_{s_{n_{k}}}(a)$. Then

$$
\left|\int_{a}^{b}\left(P_{N}(x)-1\right) d x\right| \leqq \sum_{k=1}^{\infty} \sum_{h=1}^{k}\left(\begin{array}{l}
k-1 \\
h-1
\end{array}\right) \frac{4}{n_{k}} \leqq \frac{4}{n_{1}}\left(\frac{q}{q-2}\right), \quad q>3 .
$$

LEMmA V. If $\left\{n_{k}\right\}$ is a sequence of integers with $n_{k+1} / n_{k}>q>3$ and $\nu$ $=\left(n_{k_{1}} \oplus n_{k_{2}} \oplus \cdots \oplus n_{k_{j}}\right), \quad n_{k_{1}}<n_{k_{2}}<\cdots<n_{k_{j}}$, then $\nu \in\left[n_{k_{j}}(q-2) /(q-1)\right.$, $\left.n_{k j} q /(q-1)\right]$.

Proof. It is clear that if $m=\sum_{i=0}^{\infty} x_{i} 2^{i}, n=\sum_{i=0}^{\infty} y_{i} 2^{i}, x_{i}, y_{i}=0,1$, both 
disappearing eventually, then $m-n \leqq m \oplus n \leqq m+n$. Repeating this inequality we get $n_{k_{j}}-n_{k_{j-1}}-n_{k_{j-2}}-\cdots-n_{k_{1}} \leqq n_{k_{j}} \oplus n_{k_{j-1}} \oplus \cdots \oplus n_{k_{1}} \leqq n_{k_{j}}+n_{k_{j-1}}$ $+n_{k_{j-9}}+\cdots+n_{k_{1}}$. Substituting into this expression $n_{k_{j}} / q^{r}>n_{k_{j-r}}$,

$$
\begin{gathered}
n_{k_{j}}-\frac{n_{k_{j}}}{q}-\frac{n_{k_{j}}}{q^{2}}-\cdots-\frac{n_{k_{j}}}{q^{j-1}}<\nu<n_{k_{j}}+\frac{n_{k_{j}}}{q}+\frac{n_{k_{j}}}{q^{2}}+\cdots+\frac{n_{k_{j}}}{q^{j-1}}, \\
\frac{n_{k_{j}}(q-2)}{q-1}<\nu<\frac{n_{k_{j}} q}{q-1} .
\end{gathered}
$$

After these preliminaries we proceed to the main idea of the proof. Given $n_{k+1} / n_{k}>q>1$, in general, we write $q=1+2 \epsilon$. Now choose a number $\epsilon^{\prime}>0$ which satisfies the following:

$$
\begin{array}{ll}
\text { (a) }(1+2 \epsilon)\left(1-\epsilon^{\prime}\right)>\rho>1 & \text { (b) } \frac{1+2 \epsilon}{1+\epsilon^{\prime}}>\rho>1
\end{array}
$$

$$
\text { (c) } 3\left(\frac{1-\epsilon^{\prime}}{1+\epsilon^{\prime}}\right)>\rho>1 \text {. }
$$

For this number $\epsilon^{\prime}$ find $Q\left(\epsilon^{\prime}\right)$ such that if $p>Q\left(\epsilon^{\prime}\right)$, then $(p-2) /(p-1)>1-\epsilon^{\prime}$ and $p /(p-1)<1+\epsilon^{\prime}$. Determine integer $r$ so that $q^{r}>\max \left(3, Q\left(\epsilon^{\prime}\right)\right)$.

The sequence $\left\{n_{k}\right\}$ can then be split into $r$ subsequences, $\left\{n_{k r+p}\right\}$, $p=1,2,3, \cdots, r$, with $k$ varying from 0 to $\infty$. Define also the functions

$$
\begin{aligned}
P_{N, p}(x) & =\prod_{k=0}^{N-1}\left(1+\epsilon_{k r+p} \psi(x)\right), P_{n_{k r+p}}^{(k)}(x) \\
& =\frac{P_{N, p}(x)}{\left(1+\epsilon_{k++p} \psi_{\left.n_{k r+p}(x)\right)}^{(x)}\right.}
\end{aligned}
$$

where $\epsilon_{k r+p}= \pm 1, p=1,2, \cdots, r$. Using the usual definition for the partial sum of a lacunary series, we have for each $p=1,2,3, \cdots, r$,

$$
\begin{aligned}
& \int_{a}^{b} S_{N r}(x) P_{N, p}(x) d x=\sum_{k=0}^{N-1} a_{k r+p} \int_{a}^{b}\left(1+\epsilon_{k r+p} \psi_{n_{k r+p}}^{(x)}\right) P_{N, p}^{(k)}(x) \psi\left(\underset{n_{k r+p}}{(x)} d x\right. \\
& +\sum_{p^{\prime}=1 ; p^{\prime} \neq p}^{r}\left[\sum_{k=0}^{N-1} a_{k r+p^{\prime}} \int_{a}^{b} \psi_{n_{k r+p^{\prime}}} P_{N, p}(x) d x\right] \\
& =\sum_{k=0}^{N-1} a_{k r+p} \epsilon_{k r+p}\left(\int_{a}^{b} \epsilon_{k r+p} \psi_{n_{k r+p}}^{(x)} P_{N, p}^{(k)}(x) d x\right)+\sum_{k=0}^{N-1} a_{k r+p} \epsilon_{k r+p} \int_{a}^{b} P_{N, p}^{(k)}(x) d x \\
& +\sum_{p^{\prime}=1 ; p^{\prime} \neq p}^{r} \sum_{k=0}^{N-1} a_{k r+p^{\prime}} \epsilon_{k r+p^{\prime}}\left(\int_{a}^{b} \epsilon_{k r+p^{\prime}} \psi_{n_{k r+p^{\prime}}}^{(x)} P_{N, p}(x) d x\right) \\
& =I_{1}+I_{2}+I_{3} .
\end{aligned}
$$


Consider a typical coefficient from $I_{1}$,

$$
\begin{aligned}
& \left|\int_{a}^{b} \epsilon_{k r+p} \psi{ }_{n k r+p}(x) P_{N, p}^{(k)}(x) d x\right| \\
& \qquad\left|\int_{a}^{b} \psi_{n_{k r+p}}^{(x)} d x\right|+\sum_{\nu=0 ; \nu \neq k}^{N-1}\left|\int_{a}^{b} \psi_{n_{k r+p}}^{(x)}\left(\sum_{j=0}^{\nu} G_{j v}(x)\right) d x\right|
\end{aligned}
$$

where $G_{j v}$ is the sum of all products of $(j+1) \psi$ 's, the largest subscript being $n_{\nu r+p}$. The first term on the right is majorized by $4 / n_{1}$ and the sum by

$$
\sum_{\nu=0 ; \nu \neq k}^{\infty}\left[\sum_{j=0}^{\nu}\left(\begin{array}{l}
\nu \\
j
\end{array}\right) \max _{\text {all subseripts } \gamma \text { in } G_{j \nu}}\left|\int_{a}^{b} \psi_{n k r+p}(x) \psi_{\gamma}(x) d x\right|\right] .
$$

For these integrals, because $q^{r}>\max \left[3, Q\left(\epsilon^{\prime}\right)\right]$ and $n_{s r+p} / n_{(8-1) r+p}>q^{r}$, any subscript $\gamma$ from $G_{j \nu}$ must (by Lemma $\mathrm{V}$ and choice of $Q\left(\epsilon^{\prime}\right)$ ) lie in the interval $\left[n_{\nu r+p}\left(1-\epsilon^{\prime}\right), n_{\nu r+p}\left(1+\epsilon^{\prime}\right)\right]$ where $\nu \neq k$.

But then $\gamma$ has as greatest power of 2, $2^{8 n_{v_{r}+p}}$, and $n_{k r+p}$ has a different greatest power of 2 . Their "sum" under $\oplus$ contains a power of 2 greater than or equal to $2^{s_{n_{1}+p}}$. Thus $\left|\int_{a}^{b} \psi_{n_{k r+p}}(x) \psi_{\gamma}(x) d x\right| \leqq 4 / n_{\nu r+p}$ and

$$
\left|\int_{a}^{b} \epsilon_{k r+p} \psi_{n_{k r+p}}(x) P_{N, p}^{(k)}(x) d x\right| \leqq \frac{4}{n_{1}}+\sum_{\nu=0}^{\infty} \frac{2^{v+2}}{n_{v r+p}} \leqq \frac{8}{n_{1}}\left(\frac{q^{r}}{q^{r}-2}\right), \quad q^{r}>3 .
$$

For $I_{2}$ by (7.4),

$$
\begin{aligned}
\left(\int_{a}^{b} P_{N, p}^{(k)}(x) d x\right) & =\left[\int_{a}^{b} d x-\int_{a}^{b}\left(1-P_{N, p}^{(k)}(x)\right) d x\right] \\
& \geqq(b-a)-\frac{4}{n_{1}}\left(\frac{q^{r}}{q^{r}-2}\right),
\end{aligned}
$$

Finally, for coefficients of $I_{3}$ we have

$$
\begin{aligned}
& \left|\int_{a}^{b} \epsilon_{k r+p^{\prime}} \psi_{n k r+p^{\prime}}(x) P_{N, p}(x) d x\right| \leqq\left|\int_{a}^{b} \psi_{n k r+p^{\prime}}(x) d x\right| \\
& +\sum_{\nu=0}^{N-1}\left[\sum_{j=0}^{\nu}\left(\begin{array}{l}
\nu \\
j
\end{array}\right)_{\text {all subscripts } \gamma \text { appearing } \min G_{j,}}\left|\int_{a}^{b} \psi_{n_{k r+p^{\prime}}} \underset{\gamma}{(x)} \psi_{\gamma}(x) d x\right|\right]
\end{aligned}
$$

where $p^{\prime} \neq p$, and now $\nu$ may equal $k$.

Again $\gamma$ belongs to the interval $\left[n_{\nu r+p}\left(1-\epsilon^{\prime}\right), n_{\nu r+p}\left(1+\epsilon^{\prime}\right)\right]$, and so the closest that this interval may come to $n_{k r+p^{\prime}}$ as $\nu$ and $p$ vary is when $\nu=k$ and $p=p^{\prime} \pm 1$; or $\nu=k-1$ and $p^{\prime}=1, p=r$; or $\nu=k+1$ or $p^{\prime}=r$ and $p=1$. In any of these cases $n_{\nu r+p}$ and $n_{k r+p^{\prime}}$ are succeeding terms (in some order) in the original sequence $\left\{n_{k}\right\}$. The terms on the right in (7.11) are largest when $\gamma$ and $n_{k r+p^{\prime}}$ are close. We may describe the two situations in which 
$n_{k r+p^{\prime}}$ is very close to $\gamma$, i.e., the worst possible cases as:

(a) $\gamma \in\left[n_{k}\left(1-\epsilon^{\prime}\right), n_{k}\left(1+\epsilon^{\prime}\right)\right]$ and $n_{k r+p^{\prime}}$ plays the role of $n_{k+1}$.

(b) $n_{k r+p^{\prime}}$ plays the role of $n_{k-1}$ and $\gamma \in\left[n_{k}\left(1-\epsilon^{\prime}\right), n_{k}\left(1+\epsilon^{\prime}\right)\right]$.

In the first situation the choice of $\epsilon^{\prime}$ as made at the beginning of the proof enables us to say $n_{k+1} / \gamma>n_{k+1} / n_{k}\left(1+\epsilon^{\prime}\right)>q /\left(1+\epsilon^{\prime}\right)=(1+2 \epsilon) /\left(1+\epsilon^{\prime}\right)$ $>\rho>1$. In the second case $\gamma / n_{k-1}>n_{k}\left(1-\epsilon^{\prime}\right) / n_{k-1}>q\left(1-\epsilon^{\prime}\right)=(1+2 \epsilon)$ $\cdot\left(1-\epsilon^{\prime}\right)>\rho>1$. The next lemma is easily verified.

Lemma VI. There exist constants $A$ and $B$, depending on $\rho$, such that for $m>n>A(\rho)$ and $m / n>\rho>1,(m \oplus n)$ contains a power of 2 at least as large as $2^{s_{m}-B(\rho)}$, where $m=2^{s_{m}}+m^{\prime}, 0 \leqq m^{\prime}<2^{s_{m}}$.

An application of the lemma shows that

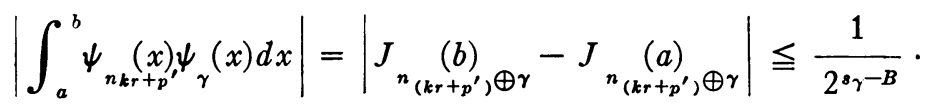

Since $\gamma$ is in the interval $\left[n_{v r+p}\left(1-\epsilon^{\prime}\right), n_{v r+p}\left(1+\epsilon^{\prime}\right)\right]$, it follows that

$$
\left|\int_{a}^{b} \psi_{n k r+p \oplus r}^{(x)} d x\right| \leqq \frac{C}{n_{v r+p}} .
$$

Using this estimate in (7.11), we have for a typical term of $I_{3}$

$$
\begin{aligned}
\left|\int_{a}^{b} \epsilon_{n_{k r+p^{\prime}}} \psi_{n_{k r+p^{\prime}}} P_{N, p}(x) d x\right| & \leqq \frac{4}{n_{1}}+\sum_{\nu=0}^{N-1}\left[\sum_{j=0}^{\nu}\left(\begin{array}{l}
\nu \\
j
\end{array}\right) \frac{C}{n_{\nu r+p}}\right] \\
& \leqq \frac{4}{n_{1}}+C \sum_{\nu=0}^{N-1} \frac{2^{v}}{n_{\nu r+p}} \\
& \leqq \frac{4}{n_{1}}\left(1+\frac{q^{r} C}{q^{r}-2}\right),
\end{aligned}
$$

Let us now observe that if $n_{1}$ is only sufficiently large, (7.9) implies that the coefficient of each term in $I_{1}$ of (7.7) is less than $\eta$ in absolute value.

Similarly, (7.10) shows that for $n_{1}$ large enough, the coefficients in $I_{2}$ of (7.7) are all non-negative and numerically larger than $[(b-a)-\eta]$. Finally, from (7.14), a large $n_{1}$ implies the coefficients in $I_{3}$ of (7.7) are all less than $\eta$ in absolute value. Setting $\epsilon_{j}=\left(\operatorname{sgn} a_{j}\right)$ we have from $(7.7)$

$$
\begin{aligned}
\int_{a}^{b} S_{N r}(x) & P_{N, p}(x) d x \\
& \geqq \sum_{k=0}^{N-1}((b-a)-2 \eta)\left|a_{k r+p}\right|-\eta \sum_{p^{\prime}=1 ; p^{\prime} \neq p}^{r} \sum_{k=0}^{N-1}\left|a_{n_{k r+p^{\prime}}}\right| .
\end{aligned}
$$

If we now sum over all $p$, 


$$
\begin{aligned}
\sum_{p=1}^{r} \int_{a}^{b} S_{N r}(x) P_{N, p}(x) d x & \geqq \sum_{j=1}^{N r}((b-a)-2 \eta)\left|a_{j}\right|-(r-1) \sum_{j=1}^{N r} \eta\left|a_{j}\right| \\
& \geqq \frac{1}{2}(b-a) \sum_{j=1}^{N r}\left|a_{j}\right|, \quad \eta<\frac{(b-a)}{2(r+1)} .
\end{aligned}
$$

However, on $[a, b],\left|S_{N r}(x)\right| \leqq M$, and since $P_{N, p}(x)$ is a product of nonnegative factors $\left(1+\psi_{n}(x)\right)$, we have

$$
\begin{aligned}
\frac{(b-a)}{2} \sum_{j=1}^{N r}\left|a_{j}\right| & \leqq \sum_{p=1}^{r} \int_{a}^{b}\left|S_{N r}(x)\right|\left|P_{N, p}(x)\right| d x \\
& \leqq M \sum_{p=1}^{r} \int_{a}^{b} P_{N, p}(x) d x \leqq M r
\end{aligned}
$$

and so as $N \rightarrow \infty$ the theorem now follows.

This result may be shown to hold under more general conditions, e.g. $S_{N}(x)$ bounded above, etc. Remarks on such generalizations are found at the close of the paper of Zygmund in which the theorem is presented. An easy consequence of this theorem is the Walsh analogue of the well known theorem of Szidon.

Theorem XXI (Szidon). If a lacunary Walsh series is the Fourier series of a function $f(x) \in B$ (class of bounded functions), then the series converges absolutely.

\section{Proof.}

$$
\begin{aligned}
& \left|S_{N}(x)-\sigma_{n_{N}}(x)\right|=\sum_{k=1}^{(N-1)} a_{k} \psi_{n_{k}}(x) \\
& -\frac{\left(n_{N}-n_{1}\right) a_{1} \psi_{n_{1}}(x)+\left(n_{N}-n_{2}\right) a_{2} \psi_{n_{2}}(x)+\cdots+\left(n_{N}-n_{N-1}\right) a_{N-1} \psi_{n_{N-1}}(x)}{n_{N}} \\
& \leqq \frac{n_{1}\left|a_{1}\right|+n_{2}\left|a_{2}\right|+\cdots+n_{N-1}\left|a_{N-1}\right|}{n_{N}} \\
& <\left(\left|a_{N-1}\right| q^{-1}+\left|a_{N-2}\right| q^{-2}+\cdots+\left|a_{1}\right| q^{(N-1)}\right) \rightarrow 0 .
\end{aligned}
$$

But from Theorem XIV, $\left\{\sigma_{n_{N}}(x)\right\}$ is a uniformly bounded sequence. It follows that $\left\{S_{N}(x)\right\}$ is a uniformly bounded sequence, and so by Theorem XX the series is absolutely convergent.

The next theorem is preliminary to establishing the analogues of the remarkable theorems of Banach on the existence of Fourier series with prescribed coefficients at lacunary places. These results are found in the text of Zygmund [21], but a less complicated presentation appears in a more recent article by Salem and Zygmund [11]. The proofs given below follow in part this more recent exposition. 
THEOREM XXII. If $\left\{n_{k}\right\}$ is any lacunary sequence of positive integers $n_{k+1} / n_{k}>q>1$ and $\left\{a_{i}\right\}$ is any sequence of real numbers tending to zero, there exists a Walsh Fourier-Stieltjes series $\sum_{j=0}^{\infty} c_{j} \psi_{j}(x)$ such that $c_{n_{i}}=a_{i}, i=1,2$, $3, \cdots$.

Proof. Suppose $\left|a_{i}\right| \leqq 1$ for all $i$. Consider first the case $n_{k+1} / n_{k}>q>3$. Since each factor is non-negative,

$$
P_{k}(x)=\prod_{i=1}^{k}\left(1+a_{i} \psi_{n_{i}}(x)\right) \geqq 0 .
$$

The following lemma proves that if $P_{k}(x)$ were multiplied out, there would be no collapsing of terms following the employment of the product formula, $\psi_{m}(x) \psi_{n}(x)=\psi_{m \oplus n}(x)$.

LEMMA VII. If $q>3$, all subscripts $\nu=\left(n_{i_{1}} \oplus n_{i_{2}} \oplus \cdots \oplus n_{i_{j}}\right), n_{i_{1}}<n_{i_{2}} \cdots$ $<n_{i_{j}}$ which appear in the expansion $P_{k}(x)=1+\sum_{\nu} \alpha_{\nu} \psi_{\nu}(x)$ are distinct.

Proof. For $q>3$, each integer $n_{i}$ necessarily contains a higher power of 2 in its dyadic expansion than its predecessor had. In comparing two distinct subscripts $\nu$, the largest "summand" to appear in the first $\nu$ is, or is not, equal to the largest "summand" to appear in the second $\nu$.

Consider $\left(n_{i_{1}} \oplus n_{i_{2}} \oplus \cdots \oplus n_{i_{j}}\right)$ and $\left(\bar{n}_{i_{1}} \oplus \bar{n}_{i_{2}} \oplus \cdots \oplus \bar{n}_{i_{p}}\right)$ in which $n_{i_{j}}>\bar{n}_{i_{p}}$. Since $n_{i_{j}}$ contains a higher power of 2 in its expansion than does $\bar{n}_{i_{p}}$, the two subscripts are distinct.

Suppose the subscripts have $n_{i_{j}}=\bar{n}_{i_{p}}$ but differ first at the $r$ th "summand." Then the one having the larger "summand" at that place will have a particular power of 2 , say $2^{s_{r}}$ (where $2^{s_{r}}$ represents the greatest power of 2 in the dyadic expansion of the $r$ th "summand") enter the calculation of its numerical value one time more than it will enter the calculation of the numerical value of the other subscript. In all cases then the subscripts are distinct, and the lemma is proved.

Hence as $k \rightarrow \infty,(7.17)$ actually becomes a Walsh series $\sum_{j=0}^{\infty} c_{j} \psi_{j}(x)$. Many $c_{j}$ may be zero since no subscript $\nu=\left(n_{i_{1}} \oplus n_{i_{2}} \oplus \cdots \oplus n_{i_{k}}\right)$ happens to equal $j$; however, each coefficient $c_{n_{i}}$ appears and has the value $a_{i}$.

Suppose $P_{k}(x)$ is the partial sum $S_{n_{k}}(x)$ of $\sum_{j=0}^{\infty} c_{j} \psi_{j}(x)$. Then $\int_{0}^{1}\left|S_{n_{k}}(x)\right| d x$ $=\int_{0}^{1}\left|P_{k}(x)\right| d x=\int_{0}^{1} P_{k}(x) d x=1$. We have therefore found a sequence of partial sums bounded in the $L^{1}$ norm. The argument which established the sufficiency statement in Theorem XVI based on the uniform boundedness in $L^{1}$ norm of $(C, 1)$ means may be repeated for a sequence of partial sums which are uniformly bounded in norm and for which the coefficients $\left\{c_{j}\right\}$ of the given series tend to zero. It is easily verified, using $\left|a_{i}\right| \leqq 1$ and $a_{i} \rightarrow 0$, that $c_{j} \rightarrow 0$ and the theorem is valid for $q>3$.

In the general case $q>1$, we break up $\left\{n_{k}\right\}$ into $r$ sequences, $\left\{n_{k}^{(1)}\right\}$, $\left\{n_{\mathfrak{k}}^{(2)}\right\}, \cdots,\left\{n_{\mathfrak{k}}^{(r)}\right\}$, in such a way that $n_{\mathfrak{k}+1}^{(s)} / n_{\boldsymbol{k}}^{(s)}>q^{r}, k=1,2, \cdots, 1 \leqq s \leqq r$ 
and $r$ being a large number which shall be defined in a moment. Let $P_{k}^{(s)}$ denote the product (7.17) corresponding to the sequence $\left\{n_{k}^{(s)}\right\}$. By Lemma $\mathrm{V}$, if $q^{r}>3$ is large enough, the series $\left[\lim _{k \rightarrow \infty} P_{k}^{(s)}\right], s=1,2, \ldots, r$ will have no overlapping. But then for this $r$ the combined series $\sum_{s=1}^{r}\left[\lim _{k \rightarrow \infty} P_{k}^{(s)}\right]$ is the required Fourier-Stieltjes series.

THEOREM XXIII (Banach). Let $\left\{n_{k}\right\}$ be any lacunary sequence of positive integers $n_{k+1} / n_{k}>q>1$ and let $\left\{a_{i}\right\}$ be any sequence of real numbers tending to zero. Then there exists a periodic function $f(x) \in L(0,1)$ such that the WalshFourier coefficients $\left\{c_{j}\right\}$ of $f(x)$ satisfy $c_{n_{i}}=a_{i}, i=1,2,3, \cdots$.

Proof. Given any sequence $\left\{a_{i}\right\}$ tending to zero and any set of positive integers $\left\{n_{k}\right\}$ there exists a convex sequence $\left\{\epsilon_{j}\right\}$ tending to zero such that $\lim _{k \rightarrow \infty}\left(a_{k} / \epsilon_{n_{k}}\right)=0$. By a result of Yano [18], the series $\sum_{k=0}^{\infty} \epsilon_{k} \psi_{k}(x)$ is a Fourier series. Since $\left(a_{k} / \epsilon_{n_{k}}\right) \rightarrow 0$, apply Theorem XXII, and there exists a Fourier-Stieltjes series $\sum_{j=0}^{\infty} p_{j} \psi_{j}(x)$ with coefficients $p_{n_{k}}=a_{k} / \epsilon_{n_{k}}$. Apply Theorem XIX, and the series $\sum_{j=0}^{\infty}\left(p_{j} \epsilon_{j}\right) \psi_{j}(x)=\sum_{j=0}^{\infty} c_{j} \psi_{j}(x)$ belongs to $L$. Then $c_{n_{k}}=\left(p_{n_{k}} \epsilon_{n_{k}}\right)=a_{k}$, and the theorem is proved.

THEOREM XXIV (Banach). Given any lacunary sequence of positive integers $\left\{n_{k}\right\}, n_{k+1} / n_{k}>q>1$, and any sequence of real numbers $\left\{a_{k}\right\}$ such that $\sum_{k=1}^{\infty} a_{k}^{2}<\infty$, there is a continuous $(W)$ function $f(x)$ on $[0,1]$ (or a continuous function $\bar{f}(\bar{x})$ on $G)$ whose W.F.S. $\sum_{j=0}^{\infty} c_{j} \psi_{j}(x)$ (or $\sum_{j=0}^{\infty} c_{j} \Psi_{j}(\bar{x})$ ) satisfies $c_{n_{k}}=a_{k}, k=1,2, \cdots$.

Proof. Given $\sum_{k=0}^{\infty} a_{k}^{2}<\infty$ and any set of positive integers $\left\{n_{k}\right\}$, there exists a convex sequence $\left\{\epsilon_{j}\right\}$ tending to zero and such that $\sum_{k=1}^{\infty} a_{k}^{2} / \epsilon_{n_{k}}^{2}<\infty$. Then $\sum_{k=0}^{\infty} \epsilon_{k} \psi_{k}(x)$ is a Fourier series. If the theorem is valid with "bounded function $f(x)$ " replacing "continuous $(W)$ function $f(x)$," an obvious application of Theorem XIX would suffice to establish the stronger result.

Lemma VIII. If a subsequence of partial sums $\left\{S_{m_{\mathrm{o}}}(x)\right\}$ of $a$ W. F. S. satisfies $\left|S_{m_{\mathrm{e}}}(x)\right| \leqq M$, the function is a bounded function.

Proof. $M^{2} \geqq \int_{0}^{1} S_{m_{e}}^{2}(x) d x=\sum_{k=0}^{m_{s}-1} a_{k}^{2}$ and hence there exists $f \in L^{2}(0,1)$ whose W.F.S. has $S_{m_{\mathrm{g}}}(x)$ as partial sums. By Lemma III, any lacunary subsequence $\left\{S_{m_{x_{t}}}(x)\right\}$ converges a.e. to $f(x)$ and $f$ is bounded a.e.

Assume $q>3$ and define

$$
\begin{aligned}
P_{\nu}(x)= & \frac{1}{i} \prod_{k=1}^{\nu}\left(1+i a_{k} \psi_{n_{k}}(x)\right)=\frac{1}{i}\left(1+i \sum a_{k} \psi_{n_{k}}(x)\right. \\
& \left.+i^{2} \sum a_{k} a_{j} \psi_{n_{k}} \oplus_{n_{j}}(x)+\cdots+i^{\nu} a_{1} \cdots a_{\nu} \psi_{n_{1}} \oplus \ldots \oplus_{n_{\nu}}(x)\right) .
\end{aligned}
$$

As in Theorem XXII, for $q>3$ all subscripts are distinct, there is no collapsing of terms, and $P_{\nu}$ is a partial sum of $P_{\nu+1}$. As $\nu \rightarrow \infty$ we obtain formally a Walsh series $\sum_{\rho=0}^{\infty} c_{\rho} \psi_{\rho}(x)$ in which some coefficients may be complex. In the natural ordering of terms (by size of subscript) and by replacing vacant 
terms by zero's, $c_{n_{k}}=a_{k}$. Now $\left|S_{n_{p+1}}(x)\right|=\left|P_{\nu}(x)\right| \leqq\left\{\prod_{k=1}^{\infty}\left(1+a_{k}^{2}\right)\right\}^{1 / 2}<+\infty$, all $x$, since $\sum_{k=1}^{\infty} a_{k}^{2}<+\infty$. Applying Lemma VIII, the series $\sum_{\rho=0}^{\infty} c_{\rho} \psi_{\rho}(x)$ is the Fourier series of a complex-valued bounded function, the real part of which satisfies the revised weaker theorem.

The case $q>1$ is handled by splitting up the original sequence $\left\{n_{k}\right\}$ into subsequences as in Theorem XXII.

The next two results are exact analogues of their classical counterparts, and since the proofs are largely repetitious of the classical case, they will not be given here.

THEOREM XXV. If $\sum_{k=1}^{\infty} a_{k}^{2}<+\infty$ and $\left\{n_{k}\right\}$ is any lacunary sequence of integers $n_{k+1} / n_{k}>g>1$, then $\sum_{k=1}^{\infty} a_{k} \psi_{n_{k}}(x)$ converges a.e. on $[0,1]$ to a function $f(x)$ which belongs to $L^{p}(0,1)$ for every $p>0$.

Theorem XXVI. If $\sum_{k=1}^{\infty} a_{k} \psi_{n_{k}}(x)$ is a lacunary Walsh series, $n_{k+1} / n_{k}$ $>q>1$, which is summable by any Toeplitz summation method in a set $E \subset[0,1]$, $|E|>0$, then $\sum_{k=1}^{\infty} a_{k}^{2}<+\infty$.

We now come to the Walsh counterpart of a Central Limit Theorem for trigonometric lacunary series as established by Salem and Zygmund [12]. The innovations are in the use of "sums" of $r$ terms in the Riesz products and in Lemma IX.

TheOREM XXVII. Let $\left\{a_{k}\right\}$ be an arbitrary sequence of real numbers satisfying

$$
\begin{gathered}
A_{N}=\left(a_{1}^{2}+a_{2}^{2}+\cdots+a_{N}^{2}\right)^{1 / 2} \rightarrow \infty \text { as } N \rightarrow \infty . \\
a_{N}=o\left(A_{N}\right) .
\end{gathered}
$$

Then for ANY lacunary sequence of positive integers $\left\{n_{k}\right\}, n_{k+1} / n_{k}>q>1$, and ANY measurable set $E \subset[0,1],|E|>0$, the distribution functions

$$
F_{N}(y: E)=\frac{\left|\left\{x \in E \mid \sum_{k=1}^{N} \frac{a_{k} \psi_{n_{k}}(x)}{A_{N}} \leqq y\right\}\right|}{|E|}, \quad N=1,2,3, \cdots
$$

converge to the Gaussian distribution with mean value zero and dispersion 1.

Proof. It is enough to show that for any $E,|E|>0$, the characteristic functions of the distributions $F_{N}(y: E)$,

$$
\begin{aligned}
\Phi_{N}(\lambda: E) & =|E|^{-1} \int_{-\infty}^{\infty} e^{i \lambda y} d F_{N}(y: E) \\
& =|E|^{-1} \int_{E} \exp \left(i \lambda \sum_{k=1}^{N} \frac{a_{k} \psi_{n_{k}}(x)}{A_{N}}\right) d x,
\end{aligned}
$$


$N=1,2,3, \cdots$, converge uniformly to $e^{-\lambda^{2} / 2}$ for $\lambda$ on any finite range.

Split the partial sum of the given lacunary series into sums of $r$ terms each so that if $N=\operatorname{Tr}+N^{\prime}, 0 \leqq N^{\prime}<r$, then

$$
\begin{aligned}
i \lambda \sum_{k=1}^{N} \frac{a_{k} \psi_{n_{k}}(x)}{A_{N}}= & i \lambda \sum_{k=0}^{T-1} \frac{\left(a_{k r+1} \psi_{n_{k r+1}}(x)+a_{k r+2} \psi_{n_{k r+2}}(x)+\cdots+a_{(k+1) r} \psi_{n(k+1) r}(x)\right)}{A_{N}} \\
& \quad+i \lambda \frac{\left(a_{T r+1} \psi_{n_{T_{r}+1}}(x)+a_{T_{r+2}} \psi_{n_{T_{r}+2}}(x)+\cdots+a_{N} \psi_{n_{N}}(x)\right)}{A_{N}} \\
& =\sum_{k=0}^{T-1} z_{N, k}+z_{N, T} .
\end{aligned}
$$

Using the $z_{N, k}$ in the formula $e^{z}=(1+z) e^{z^{2} / 2} e^{o\left(|z|^{2}\right)}$ we have

$$
\Phi_{N}(\lambda, E)
$$

(7.20)

$$
=|E|^{-1} \int_{E} \exp \left(\sum_{k=0}^{T} o\left(\left|z_{N, k}\right|^{2}\right)\right) \prod_{k=0}^{T}\left(1+z_{N, k}\right) \exp \left(\frac{1}{2} \sum_{k=0}^{T} z_{N, k}^{2}\right) d x .
$$

But $\left|z_{N, k}\right| \leqq|\lambda|\left[\left|a_{k r+1}\right|+\left|a_{k r+2}\right|+\cdots+\left|a_{(k+1) r}\right|\right] / A_{N}$ and this can be made small uniformly. For large enough $N, \sum_{k=0}^{T} O\left(\left|z_{N, k}\right|^{2}\right)$ is equivalent to

$$
\begin{aligned}
& \epsilon \sum_{k=0}^{T}\left|z_{N, k}\right|^{2} \\
& \leqq \lambda^{2} \epsilon\left[\sum_{k=0}^{T-1} \frac{\left(a_{k r+1}^{2}+a_{k r+2}^{2}+\cdots+a_{(k+1) r}^{2}\right)}{A_{N}^{2}}+\frac{\left(a_{r r+1}^{2}+\cdots+a_{N}^{2}\right)}{A_{N}^{2}}\right]
\end{aligned}
$$

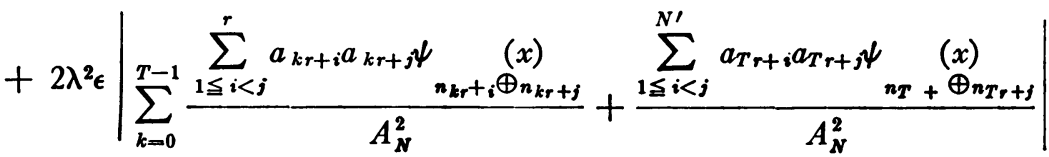

$$
\begin{aligned}
& =\epsilon\left(\lambda^{2}+2 \lambda^{2}\left|P_{N}(x)\right|\right), \epsilon \text { small. }
\end{aligned}
$$

Now $\left|P_{N}(x)\right| \leqq r(r-1)$ so that we may drop the factor $\exp \left(\sum_{k=0}^{r} o\left(\left|z_{N, k}\right|^{2}\right)\right.$ in the integrand of (7.20) with an error tending uniformly to zero.

Consider now the factor $\exp \left(1 / 2 \sum_{k=0}^{T} z_{N, k}^{2}\right)$. From (7.21)

$$
\sum_{k=0}^{T} z_{N, k}^{2}=-\lambda^{2}\left(1+2 P_{N}(x)\right) \text {. }
$$

If $\mid\left\{x \in[0,1]\left|\left\lfloor P_{N}(x) \mid \geqq \delta\right\}\right|\right.$ tends to zero as $N \rightarrow \infty$, for any $\delta>0$, it follows that $\exp \left(1 / 2 \sum_{k=0}^{T} z_{N, k}^{2}\right) \rightarrow \exp \left(-\lambda^{2} / 2\right)$ in measure, uniformly for $\lambda$ on a finite range. Then since 


$$
\begin{aligned}
&\left|\prod_{k=0}^{T}\left(1+z_{N, k}\right)\right| \leqq\left(\prod_{k=1}^{N}\left(1+\left|z_{N, k}\right|^{2}\right)\right)^{1 / 2} \\
& \leqq \exp \left(\sum_{k=0}^{T}\left|z_{N, k}\right|^{2}\right) \leqq M(\lambda), x \in[0,1], \\
&\left.\left|\Phi_{N}(\lambda, E)-\right| E\right|^{-1} e^{-\lambda^{2} / 2} \int_{E} \prod_{k=0}^{T}\left(1+z_{N, k}\right) d x \mid
\end{aligned}
$$$$
\rightarrow 0 \text { uniformly for } \lambda=O(1) \text {. }
$$

For any $\delta>0,\left|\left\{x \in[0,1]|| P_{N}(x) \mid \geqq \delta\right\}\right| \leqq \int_{0}^{1} P_{N}^{2}(x) / \delta^{2} d x$. By expanding,

$$
\begin{aligned}
& \int_{0}^{1} \frac{P_{N}^{2}(x)}{\delta^{2}} d x=\frac{1}{\delta^{2}} \int_{0}^{1} \frac{1}{A_{N}^{4}}\left\{\sum _ { k = 0 } ^ { T - 1 } \left(\sum_{1 \leqq i<j}^{r} a_{k r+i} a_{k r+j} \psi_{n_{k r+i} \oplus^{n k r+j}}(x)\right.\right. \\
& \left.+\sum_{1 \leqq i<j}^{N^{\prime}} a_{T r+i} a_{T r+j} \psi_{n T r+i \oplus \oplus^{n T r+j}}\right\}^{2} d x \\
& =\frac{1}{\delta^{2} A_{N}^{4}}\left\{\sum_{k=0}^{T-1}\left(\sum_{1 \leqq i<j}^{r} a_{k r+i}^{2} a_{k r+j}^{2}\right)+\sum_{1 \leqq i<j}^{N \prime} a_{T r+i}^{2} a_{T r+j}^{2}\right\}
\end{aligned}
$$

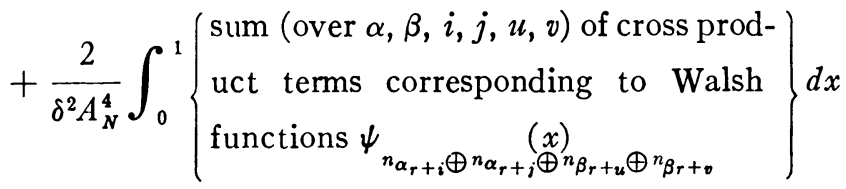

where in the last term $i, j, u, v \leqq r$, and $0 \leqq \alpha \leqq \beta \leqq T$; if $\alpha=\beta$, then $i \neq u$ or $j \neq v$.

The terms involving squares are easily dealt with as

$$
\begin{aligned}
& \frac{1}{A_{N}^{4}}\left\{\sum_{k=0}^{T-1}\left(\sum_{1 \leqq i<j}^{r} a_{k r+i}^{2} a_{k r+j}^{2}\right)+\sum_{1 \leqq i<j}^{N \prime} a_{r+i}^{2} a_{T r+j}^{2}\right\} \\
& \leqq \frac{r(r-1)}{2} \sum_{k=0}^{T-1} \frac{\max _{1 \leqq i \leqq r}\left|a_{k r+i}\right|^{4}}{A_{N}^{4}}+\frac{N^{\prime}\left(N^{\prime}-1\right)}{2} \frac{\max _{1 \leqq i \leqq N}\left|a_{T r+i}\right|^{4}}{A_{N}^{4}} \\
& \leqq \frac{r(r-1)}{2} \frac{\sum_{i=1}^{N}\left|a_{i}\right|^{4}}{A_{N}^{4}} \rightarrow 0 .
\end{aligned}
$$

The remaining terms can be separated into two classes; class $A_{1}$, those for

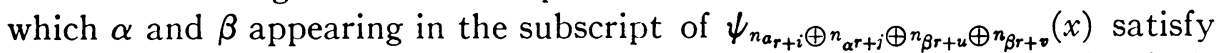
$\beta-\alpha \geqq 2$ and class $A_{2}$, those for which $0 \leqq \beta-\alpha \leqq 1$. Class $A_{2}$ can be dealt with by estimating the number of terms in the class. As $\alpha$ and $\beta$ vary from 0 to $T$, there are $T$ pairs $(\alpha, \beta)$ with $\beta-\alpha=1$ and $(T+1)$ pairs $\alpha=\beta$. The num- 
ber of terms for a given $\alpha, \beta$ with $\alpha=\beta$ is not more than $(r(r-1) / 2)^{2}$. Thus the total number of terms in class $A_{2}$ does not exceed $2(T+1)(r(r-1) / 2)^{2}$, and

$$
\begin{aligned}
\mid \int_{0}^{1}\left\{\text { terms of class } A_{2}\right\} d x \mid & \leqq 4 \int_{0}^{1}\left(\frac{r(r-1)}{2}\right)^{2}\left[\frac{\sum_{\alpha=0}^{T} \max _{1 \leqq i \leqq r}\left|a_{\alpha r+i}\right|^{4}}{A_{N}^{4}}\right] d x \\
& \leqq 4\left(\frac{r(r-1)}{2}\right)^{2} \frac{\sum_{k=1}^{N}\left|a_{h}\right|^{4}}{A_{N}^{4}} \rightarrow 0 .
\end{aligned}
$$

It remains only to show that the sum of the terms in class $A_{1}$ is small.

Consider a typical term of $A_{1}$,

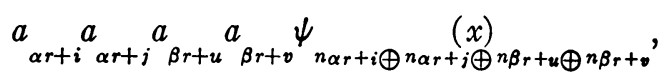

where $\beta-\alpha \geqq 2,1 \leqq i<j \leqq r, 1 \leqq u<v \leqq r$. It follows that $\alpha r+j \leqq(\beta r+u)-r$, and so there are at least $r$ numbers of the given sequence $\left\{n_{k}\right\}$ between $n_{\alpha r+j}$ and $n_{\beta r+u}$. Therefore $n_{\beta r+v} / n_{\alpha r+j}>q^{r}$. We cannot claim that for the $\psi$ in (7.28), $\int_{0}^{1} \psi d x=0$ since $\psi$ may be identically 1 . That this does not happen too frequently is the gist of the following basic lemma.

LEMMA IX. Let $\left\{n_{k}\right\}$ be any lacunary sequence of positive integers $n_{k+1} / n_{k}$ $>q>1$. For this $q$ there exists a value $T(q)$ such that if $r>T(q)$, then for each $\gamma=0,1,2, \cdots$ the equation $n_{k_{1}} \oplus n_{k_{2}} \oplus \cdots \oplus n_{k_{p}}=\gamma, n_{k_{1}}>n_{k_{2}}>\cdots>n_{k_{p}}$, has a finite number of solutions when $n_{k_{1}} / n_{k_{3}}>q^{r}$; in fact, there exists for each $\gamma$ and this $q$ a constant $C(\gamma, q)$ such that the number of solutions is less than $C^{p}(\gamma, q)$.

Proof. Write $\gamma=\sum_{i=0}^{s_{\underline{\gamma}}} m_{i} 2^{i}, m_{i}=0,1, m_{s_{\gamma}}=1$. Then by the definition of $\oplus$, the equation $n_{k_{1}} \oplus n_{k_{2}} \oplus \cdots \oplus n_{k_{p}}=\gamma$ may be written in the tabular form:

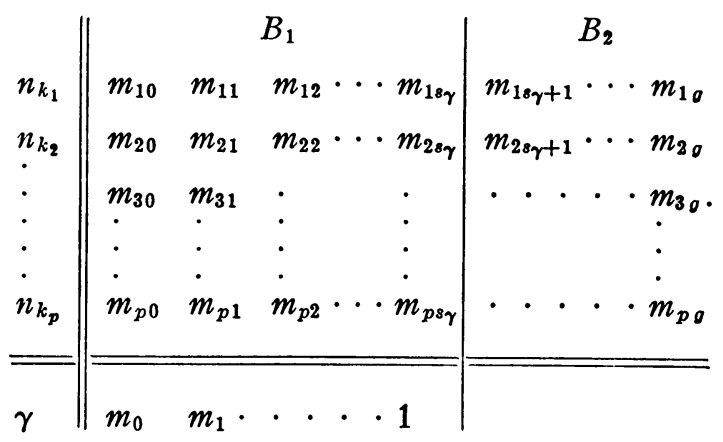

Here $n_{k_{j}}=\sum_{i=0}^{o} m_{j i} 2^{i}, j=1,2, \cdots, p$, where $g$ is the largest exponent of 2 with nonzero coefficient in any $n_{k_{i}}$. Now the entries in box $B_{1}$ must add up 
column-wise to $m_{i}(\bmod 2)$, while the entries in $B_{2}$ must add column-wise to zero $(\bmod 2)$ if the "sum" is to equal $\gamma$.

Let $j$ be an integer for which $1+1 / 2^{j} \leqq q$. Using $s_{b}$ to denote the largest power of 2 contained in the integer $b$, we have for large $n_{k_{1}}>\gamma, 2^{s} n_{k_{1}}=2^{o}>2^{s} \gamma$ and $n_{k_{1}} / n_{k_{3}} \leqq 2^{0+1-\beta}$, where $s_{n_{k_{3}}}=\beta$. Since $q>1, q^{r \rightarrow \infty}$ as $r \rightarrow \infty$, and hence $r$ may be chosen so that if $n_{k_{1}} / n_{k_{3}}>q^{r}$ is to hold, then $(g+1-\beta)$ must be large. This means (since the entries in $B_{2}$ must add to zero column-wise (mod 2)) that $n_{k_{2}}$ is not large enough, does not extend far enough into $B_{2}$ to help cancel out the upper dyadic entries of $n_{k_{1}}$. Hence $n_{k_{2}}$ and $n_{k_{1}}$ must be identical in the upper dyadic entries, that is, in the last $(g+1-\beta)$ entries. If $n_{k_{1}}$ does not have $(g+1-\beta)$ dyadic entries past $2^{2} \gamma$, then $n_{k_{1}}$ and $n_{k_{2}}$ have identical entries in all places past the $s_{\gamma}$ th power of 2, i.e., all entries in $B_{2}$. In the extreme case when $n_{k_{1}}$ and $n_{k_{2}}$ are identical in all the upper $(g+1-\beta)$ entries then

$$
\begin{aligned}
\frac{n_{k_{1}}}{n_{k_{2}}} & \leqq \frac{\left(2^{\sigma}+m_{o-1} 2^{o-1}+\cdots+m_{\beta+1} 2^{\beta+1}+m_{\beta} 2^{\beta}\right)+\left(2^{\beta-1}+2^{\beta-2}+\cdots+2^{0}\right)}{\left(2^{\sigma}+m_{g-1} 2^{o-1}+\cdots+m_{\beta+1} 2^{\beta+1}+m_{\beta} 2^{\beta}\right)} \\
& \leqq 1+\frac{2^{\beta}}{2^{\sigma}}=1+\frac{1}{2^{\sigma-\beta}} .
\end{aligned}
$$

If $(g-\beta) \geqq j$, we see that $n_{k_{1}} / n_{k_{2}} \leqq 1+1 / 2^{j} \leqq q$, which, of course, cannot be.

All this implies that by taking $r$ large enough, we can insure that in any solution of $n_{k_{1}} \oplus n_{k_{2}} \oplus n_{k_{3}} \oplus \cdots \oplus n_{k_{p}}=\gamma$ with large $n_{k_{1}}, n_{k_{1}}$ and $n_{k_{2}}$ are identical in all the upper $g-\beta \geqq j$ entries if these all lie in $B_{2}$. But then $n_{k_{1}} / n_{k_{2}}<q$, and this cannot be. The conclusion is that for such a choice of $r$, all solutions of $n_{k_{1}} \oplus n_{k_{2}} \oplus \cdots \oplus n_{k_{p}}=\gamma$ must have some of the $(g-\beta)$ upper entries of $n_{k_{1}}$ lying in $B_{1}$ if the sequence $\left\{n_{k}\right\}$ is lacunary. But then any such solution is found among those sets of integers $n_{k_{1}}>n_{k_{2}}>\cdots>n_{k_{p}}$ for which $n_{k} \leqq 2^{s} \gamma^{+i}$. The number of such solutions is clearly less than $\left[2^{8} \gamma^{+i}\right]^{p}=C(\gamma, q)^{p}$.

Apply Lemma IX with $p=4$ and $\gamma=0$ to the terms in class $A_{1}$ of (7.25) and obtain $r$ such that

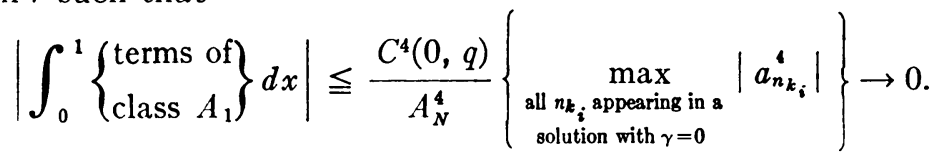

Combining (7.26), (7.27), and (7.30), it is clear that the integral $\int_{0}^{1} P_{N}^{2}(x) / \delta^{2} d x$ $\rightarrow 0$ for each $\delta$ as $N \rightarrow \infty$, and so (7.24) has been established.

Now $\prod_{k=0}^{T}\left(1+z_{N, k}\right)=\alpha_{0}^{(N)}+\sum \alpha_{\gamma}^{(N)} \psi_{\gamma}(x)$ where $\alpha_{\gamma}^{(N)}$ is the sum of all coefficients in the expansion whose subscripts correspond to a solution $n_{k_{1}} \oplus n_{k_{2}}$ $\oplus \cdots \oplus n_{k_{p}}=\gamma, n_{k_{1}}>n_{k_{2}}>\cdots>n_{k_{p}}$, for $p=1,2,3, \cdots, N$. In performing the multiplication above, it is noticed that $n_{k_{1}}, n_{k_{2}}$, and $n_{k_{3}}$ in any sum for $\gamma$ must necessarily come from three different sums of $r$ terms, i.e., three different $z_{N, k}$ 's. Hence $n_{k_{1}}$ and $n_{k_{z}}$ have at least $r$ of the integers $\left\{n_{k}\right\}$ between them. Thus $n_{k_{1}} / n_{k_{3}}>q^{r}$, and by Lemma IX 


$$
\left|\alpha_{\gamma}^{(N)}\right| \leqq \sum_{p=1}^{N}|\lambda|^{p} C^{p}(\gamma, q) \max _{1 \leqq i \leqq N}\left|a_{i}\right|^{p} / A_{N}^{p} .
$$

But for $N$ large, $\left(C|\lambda| \max _{1 \leqq i \leqq N}\left|a_{i}\right|\right) / A_{N}<\epsilon / 2$ uniformly for $\lambda$ on a finite range so that $\left|\alpha_{\gamma}^{(N)}\right| \leqq \epsilon$. This holds also for $\left|\alpha_{0}^{(N)}-1\right|$ and so

$$
\alpha_{\gamma}^{(N)} \rightarrow 0, \gamma=1,2,3, \cdots \text { and } \alpha_{0}^{(N)} \rightarrow 1 \text {, uniformly for } \lambda=0(1),
$$

$\int_{E} \prod_{k=0}^{T}\left(1+z_{N, k}\right) d x \rightarrow|E|$ uniformly for $\lambda$ on a finite range, and the theorem is proved.

8. Fourier-Stieltjes coefficients. In this final section an example will be given of a continuous function of bounded variation whose Walsh FourierStieltjes coefficients do not tend to zero. Hille and Tamarkin [6] have calculated the trigonometrical Fourier-Stieltjes coefficients of the classical Lebesgue function $\omega(x)$ (a function based on the Cantor ternary set) which is continuous and of bounded variation and have shown an infinite number of the coefficients to be bounded away from zero. The Cantor set may be generalized by dropping out (at each step in the construction) subintervals the ratio of whose measures to that of the containing subinterval is an arbitrary value $\theta, 0<\theta<1$. The resulting Lebesgue functions $\omega_{\theta}(x)$ constructed on these generalized sets are each continuous and of bounded variation. The Walsh Fourier-Stieltjes coefficients of the Lebesgue function constructed with $\theta=1 / 2$ do not tend to zero. This example establishes the next theorem.

Theorem XXVIII. The Walsh Fourier-Stieltjes coefficients of continuous function of bounded variation need not tend to zero.

Proof. On the interval $[0,1]$ drop out the open interval $(1 / 4,3 / 4)$. From $[0,1 / 4]$ drop out the open interval $(1 / 16,3 / 16)$; from $[3 / 4,1]$ drop out $(13 / 16,15 / 16)$, etc. At the $n$th step drop out from the remaining $2^{n-1}$ closed intervals open subintervals each of length $1 / 2$ of the containing interval and centrally located within the same. The set of points which remain, when this is done infinitely often, is the Cantor set of ratio $1 / 2$. It consists of all endpoints of all of the closed intervals used during the construction and all their limit points. It may be verified that, just as the classical Cantor set consists of all points $x=\sum_{i=1}^{\infty} a_{i} / 3^{i}, a_{i}=0$ or 2 , so the Cantor set of ratio $1 / 2$ consists precisely of all points $x=\sum_{i=1}^{\infty} a_{i} / 4^{i}, a_{i}=0$ or 3 .

Let $\omega(x) \equiv \omega_{1 / 2}(x)$ be defined by specifying that

$$
\omega(x)=\sum_{i=1}^{\infty} \frac{b_{i}}{2^{i}} \quad \text { where } \quad x=\sum_{i=1}^{\infty} \frac{a_{i}}{4^{i}}, \quad a_{i}=0 \text { or } 3, \text { and } b_{i}=\frac{a_{i}}{3},
$$

if $x \in C$,

$\omega(x)=$ common value of $\omega(x)$ at the end points of the open interval of the complement of $C$ in which $x$ lies,

if $x \notin C$. 
$\omega(x)$ is continuous, monotone, nondecreasing, and constant on each of the subintervals rejected at some stage in the construction of $C$.

The Walsh Fourier-Stieltjes coefficients of $\omega(x)$ are defined by $\alpha_{k}$ $=\int_{0}^{1} \psi_{k}(x) d \omega(x)$. This Riemann-Stieltjes integral exists and hence for any partitions $P_{t}=\left\{0=x_{0}<x_{t, 1}<x_{t, 2}<\cdots<x_{t, m_{t}}=1\right\}$, of norms tending to zero and any $\xi_{t, i} \in\left[x_{t, i-1}, x_{t, i}\right)$,

$$
\alpha_{k}=\lim _{t \rightarrow \infty} \sum_{i=1}^{m_{t}} \psi_{k}\left(\xi_{t, i}\right)\left[\omega\left(x_{t, i}\right)-\omega\left(x_{t, i-1}\right)\right] .
$$

After the $t$ th step in the construction of $C$ there remain $2^{t}$ closed intervals $A_{j}^{(t)}, j=1,2, \cdots, 2^{t}$, while $2^{t-1}$ open intervals have just been removed. Formula (8.1) implies that on any of the closed intervals $\omega(x)$ increases by $1 / 2^{t}$. We therefore have

$$
\alpha_{k}=\lim _{t \rightarrow \infty} \sum_{s=1}^{2^{t}} \frac{\psi_{k}\left(\xi_{s}\right)}{2^{t}},
$$

Consider the integers $\left\{\left(2^{2 n}+2^{2 n+1}\right)\right\}, n=1,2,3, \cdots$. Any Walsh function of the form

$$
\psi_{k}(x)=\psi_{2^{2 n}+2^{2 n-1}(x)}=\phi_{2 n}(x) \phi_{2 n+1}(x)
$$

has a symmetric graph with respect to the abscissa $1 / 2$. The corresponding coefficient (8.2) takes the form

$$
\alpha_{k}=2 \lim _{t \rightarrow \infty} \sum_{s=1}^{2^{t-1}} \frac{\psi_{k}\left(\xi_{s}\right)}{2^{t}}
$$

where $\xi_{s}$ is any number in $A_{s}^{(t)}$. Choose $\xi_{s}$ as the left-hand end-point of $A_{s}^{(t)}$. The left end-points of the interval $A_{s}^{(t)}$ have dyadic expansions of the form

$$
\xi_{s}=\frac{\alpha_{1}}{2}+\frac{\alpha_{1}}{2^{2}}+\frac{\alpha_{2}}{2^{3}}+\cdots+\frac{\alpha_{t}}{2^{2 t-1}}+\frac{\alpha_{t}}{2^{2 t}}+0+0+\cdots, \quad \alpha_{i}=0,1 .
$$

For the special sequence of integers $k=2^{2 n}+2^{2 n+1}$ and the special points $\xi_{8}$ we have $\psi_{k}\left(\xi_{s}\right)=\phi_{2 n}\left(\xi_{s}\right) \phi_{2 n+1}\left(\xi_{s}\right)=+1$ since the $(2 n+1)$ st and $(2 n+2)$ nd places in the dyadic expansion of $\xi_{s}$ are identical. Thus for this sequence of values of $k(8.3)$ implies $\alpha_{k}=2 \lim _{t \rightarrow \infty} \sum_{s=1}^{2^{t-1}} 1 / 2^{t}=1$.

REMARK. It can easily be shown that the Walsh Fourier-Stieltjes coefficients of the generalized Lebesgue function $\omega_{\theta}(x)$ are given by

$$
\begin{aligned}
& \alpha_{0}(\theta)=1, \\
& \int=0 \text { if } k \text { has an odd number of nonzero terms in its dyadic expansion, } \\
& \alpha_{k}(\theta)\left\{=\left[1-\sum_{i=1}^{2^{s} k^{+1}-1} \omega_{\theta}\left(\frac{i}{2^{s_{k}+1}}\right)\left\{\psi_{k}\left(\frac{2 i+1}{2^{s_{k}+2}}\right)-\psi_{k}\left(\frac{2 i-1}{2^{s_{k}+2}}\right)\right\}\right]\right. \text { otherwise. }
\end{aligned}
$$




\section{BIBLIOGRAPHY}

1. N. J. Fine, On the Walsh functions, Trans. Amer. Math. Soc. vol. 65 (1949) pp. 372414. 66-77.

2. - The generalized Walsh functions, Trans. Amer. Math. Soc. vol. 69 (1950) pp.

3. - Cesdro summability of Walsh-Fourier series, Proc. Nat. Acad. Sci. U.S.A. vol. 41 (1955) pp. 588-591.

4. Paul R. Halmos, Measure theory, New York, 1950.

5. T. Hildebrandt, Stieltjes integrals of the Riemann type, Amer. Math. Monthly, vol. 45 (1938) pp. 265-278.

6. E. Hille and J. D. Tamarkin, Remarks on a known example of a monotone continuous function, Amer. Math. Monthly vol. 36 (1929) pp. 255-264.

7. J. Marcinkiewicz and A. Zygmund, On the differentiability of functions and summability of trigonometrical series, Fund. Math. vol. 26 (1936) pp. 1-43.

8. W. Orlicz, Beiträge zur Theorie der Orthogonalentwicklungen, Studia Mathematica vol. 1 (1929) pp. 1-39.

9. R. E. A. C. Paley, A remarkable series of orthogonal functions, Proc. London Math. Soc. vol. 34 (1932) pp. 241-279.

10. H. A. Rademacher, Einige Sätze über Reihen von allgemeinen Orthogonalfunctionen, Math. Ann. vol. 87 (1922) pp. 112-138.

11. R. Salem and A. Zygmund, On a theorem of Banach, Proc. Nat. Acad. Sci. U.S.A. vol. 33 (1947) pp. 293-295.

12. - On lacunary trigonometric series, Proc. Nat. Acad. Sci. U.S.A. vol. 33 (1947) pp. 333-338.

13. A. A. Sneider, On series of Walsh functions with monotone coefficients, Bull. Acad. Sci. URSS. Sér. Math. vol. 12 (1948) pp. 179-192.

14. - On the uniqueness of expansions in Walsh functions, Rec. Math. (Mat. Sbornik) N.S. vol. 24 (66) (1949) pp. 279-300.

15. - On the convergence of subsequences of the partial sums of Fourier series of Walsh functions, C. R. (Doklady) Acad. Sci. URRS. vol. 70 (1950) pp. 969-971.

16. Gen-Ichirô Sunouchi, On the Walsh-Kaczmarz series, Proc. Amer. Math. Soc. vol. 2 (1951) pp. 5-11.

17. N. Vilenkin, On a class of complete orthonormal systems, Bull. Acad. Sci. URSS. Sér. Math. vol. 11 (1947) pp. 363-400.

18. Shigeki Yano, On Walsh-Fourier series, Tôhoku Math. J. vol. III (1951) pp. 223-242.

19. - On approximation by Walsh functions, Proc. Amer. Math. Soc. vol. 2 (1951) pp. 962-967.

20. A. Zygmund, Quelques théorèmes sur les séries trigonométriques et celles de puissances, Studia Mathematica vol. 3 (1931) pp. 77-83.

21. - Trigonometrical series, Warsaw, 1935.

University of Chicago, Chicago, Ill. 